\title{
ASYMPTOTIC INDEPENDENCE AND A NETWORK TRAFFIC MODEL
}

\author{
KRISHANU MAULIK, SIDNEY RESNICK, AND HOLGER ROOTZÉN
}

\begin{abstract}
The usual concept of asymptotic independence, as discussed in the context of extreme value theory, requires the distribution of the coordinatewise sample maxima under suitable centering and scaling to converge to a product measure. However, this definition is too broad to conclude anything interesting about the tail behavior of the product of two random variables, which are asymptotically independent. Here we introduce a new concept of asymptotic independence, which allows us to study the tail behavior of products. We carefully discuss equivalent formulations of asymptotic independence. We then use the concept in the study of a network traffic model. The usual infinite source Poisson network model assumes sources begin data transmissions at Poisson time points and continue for random lengths of time. It is assumed that the data transmissions proceed at a constant, non-random rate over the entire length of the transmission. However, analysis of network data suggests that the transmission rate is also random with regularly varying tail. So, we modify the usual model to allow transmission sources to transmit at a random rate over the length of the transmission. We assume the rate and the time have finite mean, infinite variance and possess asymptotic independence, as defined in the paper. We finally prove a limit theorem for the input process showing that the centered cumulative process under a suitable scaling converges to a totally skewed stable Lévy motion in the sense of finite dimensional distributions.
\end{abstract}

\section{INTRODUCTION}

There have been various attempts made in the literature to weaken the condition of independence of two random variables. One such notion is that of asymptotic independence used in the context of the extreme value theory [cf. 22, Chapter 5]. The joint distribution of two random variables is asymptotically independent if the coordinatewise maximum of $n$ iid observations from that distribution under suitable scaling has a non-degenerate limit which is a product measure, as $n$ increases to $\infty$. However, as shown with examples in Section 2, this concept is too weak to conclude anything meaningful about the product of the random variables. So we introduce a new concept of asymptotic independence, which is stronger than the asymptotic independence used in extreme value theory, but weaker than independence. This concept of asymptotic independence is not symmetric. We study this concept and its equivalent formulation in Section 2. Under additional moment conditions, we study the behavior of the product of two asymptotically independent random variables in Section 3 and give some illuminating examples. Then we use this concept of asymptotic independence to study a network traffic model. This network traffic model motivated our study of asymptotic independence and products since the product of transmission rate and transmission duration yields the quantity transmitted.

Long range dependence, self-similarity and heavy tails are established concepts required for modeling broadband data networks. This is especially true when analyzing internet data, as described

1991 Mathematics Subject Classification. Primary 90B15; secondary 60K250.

Key words and phrases. asymptotic independence, $M / G / \infty$ queue, network modeling, infinite source Poisson model, heavy tails, long range dependence, random rates, Poisson input .

Sidney Resnick and Krishanu Maulik were supported by NSF Grant 0071073 at Cornell University. 
in, for example, Willinger and Paxson [30]. The inadequacy of the finite variance model and short range dependence is well documented [cf. 6, 17, 31].

Network traffic models generally contain many sources transmitting data. Transmissions can be modelled by the infinite source Poisson model, sometimes called the M/G/ $\infty$ input model [710, 14, 19, 21]. The times between the starts of transmissions are modelled as iid exponentially distributed random variables. Thus, to account for the long range dependence and self-similar nature of the traffic, it becomes important to consider transmission times to be heavy tailed [32].

Most of the existing research assumes the rate of transmission to be constant and non-random. Konstantopoulos and Lin [11] replace the constant, non-random rate by a deterministic rate function which is regularly varying. They show that the cumulative input process at a large time scale is approximated by a stable Lévy motion. Resnick and van den Berg [21] extended the convergence to hold on the space $D[0, \infty)$ of càdlàg functions with Skorohod's $M_{1}$ topology [cf. 23, 26-29]. A recent empirical study on several internet traffic data sets by Guerin et al. [7] shows that the infinite source Poisson model often gives an inadequate fit to data. This study suggests that the transmission rate is also a random variable with a heavy tail. There have been few studies of this aspect of the internet traffic data modeling. In a series of recent papers, Levy, Pipiras and Taqqu [cf. 12, 15, 16] consider the case where the transmission rate is also random for a superposition of renewal reward processes. They show that the limiting behavior for large time scale and large number of superpositioned models can either be a stable Lévy process with stationary, independent increments or symmetric stable process with stationary, but dependent increments, depending on the relative rate of growth of the time scale and number of models. Their results parallel the results of Mikosch et al. [14] for the infinite source Poisson model who also obtain two different limits depending on the growth rate time scale relative to the intensity of the Poisson process.

The Levy, Pipiras, Taqqu papers mentioned above consider the renewal-reward model and assume the transmission rate to be independent of the length of transmission. It is difficult to conclude from evidence in measured data that rate and the length of the transmission are always independent, but in certain cases we may reasonably assume that the rate and the length of the transmission are at least asymptotically independent. As an example, we consider the BUburst dataset considered by Guerin et al. [7]. This is data processed from the original 1995 Boston University data described in the report by Cunha et al. [4] and cataloged at the Internet Traffic Archive (ITA) web site www. acm.org/sigcomm/ITA/. A plot of the transmission length against the transmission rate, (see Figure 1) shows that most of the data pairs hug the axes, which suggests the variables are at least asymptotically independent. However, if we plot the data in the log scale on both the axes, then a weak linear dependence is observable and the correlation coefficient between the two variables after $\log$ transform is approximately -0.379 , which argues against an independence assumption. We consider the log transform to make the variables have finite second moment, so that the correlation coefficient becomes meaningful.

The Hill estimates obtained for the transmission length, the transmission rate and the size of the transmitted file are $1.407,1.138$ and 1.157 respectively. These estimates are consistent with the observations made in Guerin et al. [7]. The corresponding Hill plots are given in Figure 2. For each of the variables, the plots in the first column, named Hill plot, give plots of $\left\{\left(k, \hat{\alpha}_{k, n}\right): 1 \leqslant\right.$ $k \leqslant n\}$, where $\hat{\alpha}_{k, n}$ is the Hill estimator of $\alpha$ based on $k$ upper order statistics. The plots in the second column, named AltHill plot [cf. 18], give the Hill estimates in an alternative scale and plot $\left\{\left(\theta, \hat{\alpha}_{\left[n^{\theta}\right\rceil, n}\right): 0 \leqslant \theta \leqslant 1\right\}$ [cf. 18]. This plot expands the original Hill plot on the left side and helps looking at that part more closely. The third plot, named the Stărică plot, is an exploratory device suggested by Stărică [24, Section 7] to decide on the number of upper order statistics to be 

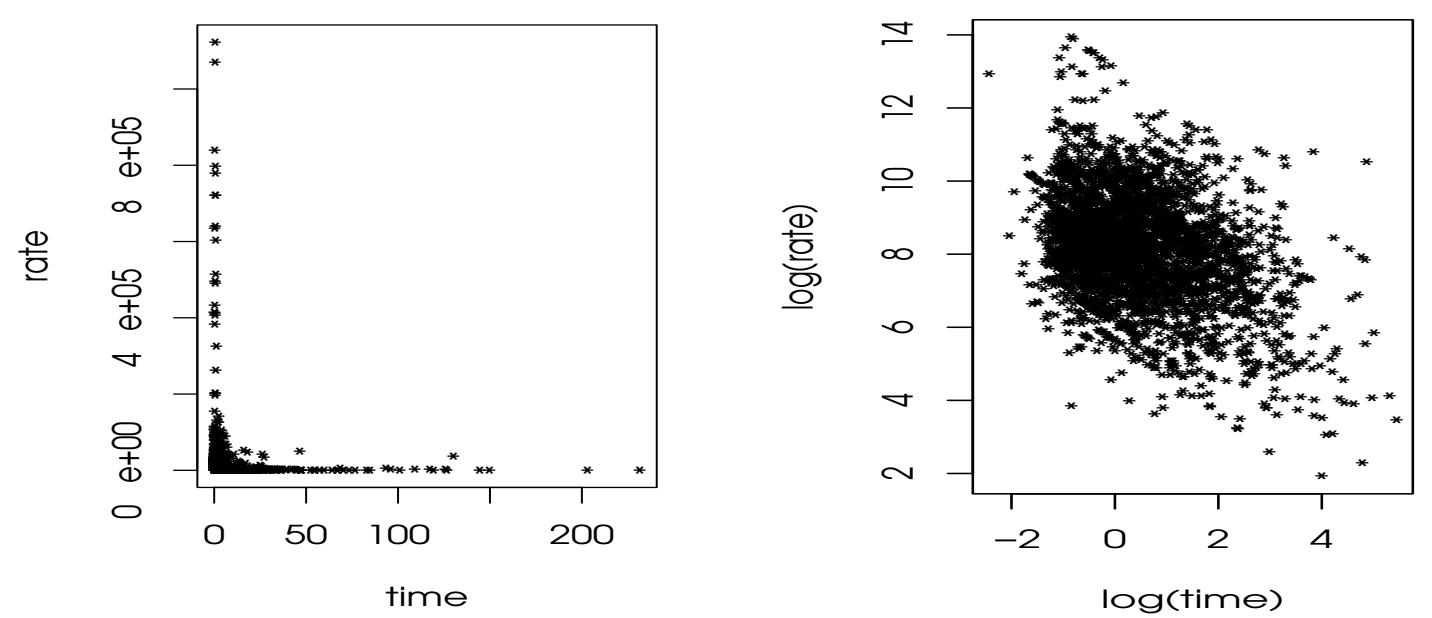

Figure 1. Plot of the time of transmission against the rate of the transmission of the BUburst data: left) in natural scale, right) in log-log scale

used. It uses the fact that for a random variable $X$ with Pareto tail of parameter $\alpha$, we have

$$
\lim _{T \rightarrow \infty} T \mathrm{P}\left[\frac{X}{T^{\frac{1}{\alpha}}}>r\right]=r^{-\alpha} .
$$

For every $k$, we estimate the left hand side by

$$
\hat{\nu}_{n, k}((r, \infty])=\frac{1}{k} \sum_{i=1}^{n} \mathbf{I}\left[\frac{X_{i}}{(n / k)^{1 / \hat{\alpha}_{n, k}}}>r\right] .
$$

We expect the ratio of $\hat{\nu}_{n, k}((r, \infty])$ and $r^{-\hat{\alpha}_{n, k}}$, called the scaling ratio, to be approximately 1 , at least for values of $r$ in a neighborhood of 1 , if we have made the correct choice of $k$. In the Starica plot, we plot the above scaling ratio against the scaling constant $r$, and choose $k$ so that the graph hugs the horizontal line of height 1 . The interesting point to be noted is the fact that the rate of the transmission has a much heavier tail than the length of transmission. This justifies the study of a model with a random rate with heavy tails. The tail of the size of the transmitted file, which is the product of the rate and the time of transmission, is comparable to the rate of the transmission, the heavier one between time and rate. This is in agreement with Theorem 3.1.

Since both the transmission length and the transmission rate have marginal distributions with heavy tails, it is further reasonable to assume that their bivariate distribution has a bivariate regularly varying tail, which is asymptotically independent in the sense used in extreme value theory. However, as described in Sections 2 and 3, the usual notion of asymptotic independence from extreme value theory is not sufficient for meaningful analysis. So we assume that the transmission and the transmission length are assumed to be asymptotically independent in the sense described in Section 2. Section 4 outlines the network model and states the limit theorem, which is proved in Section 5. Section 6 comments on the appropriateness of the model for the data and suggests possible improvements. 

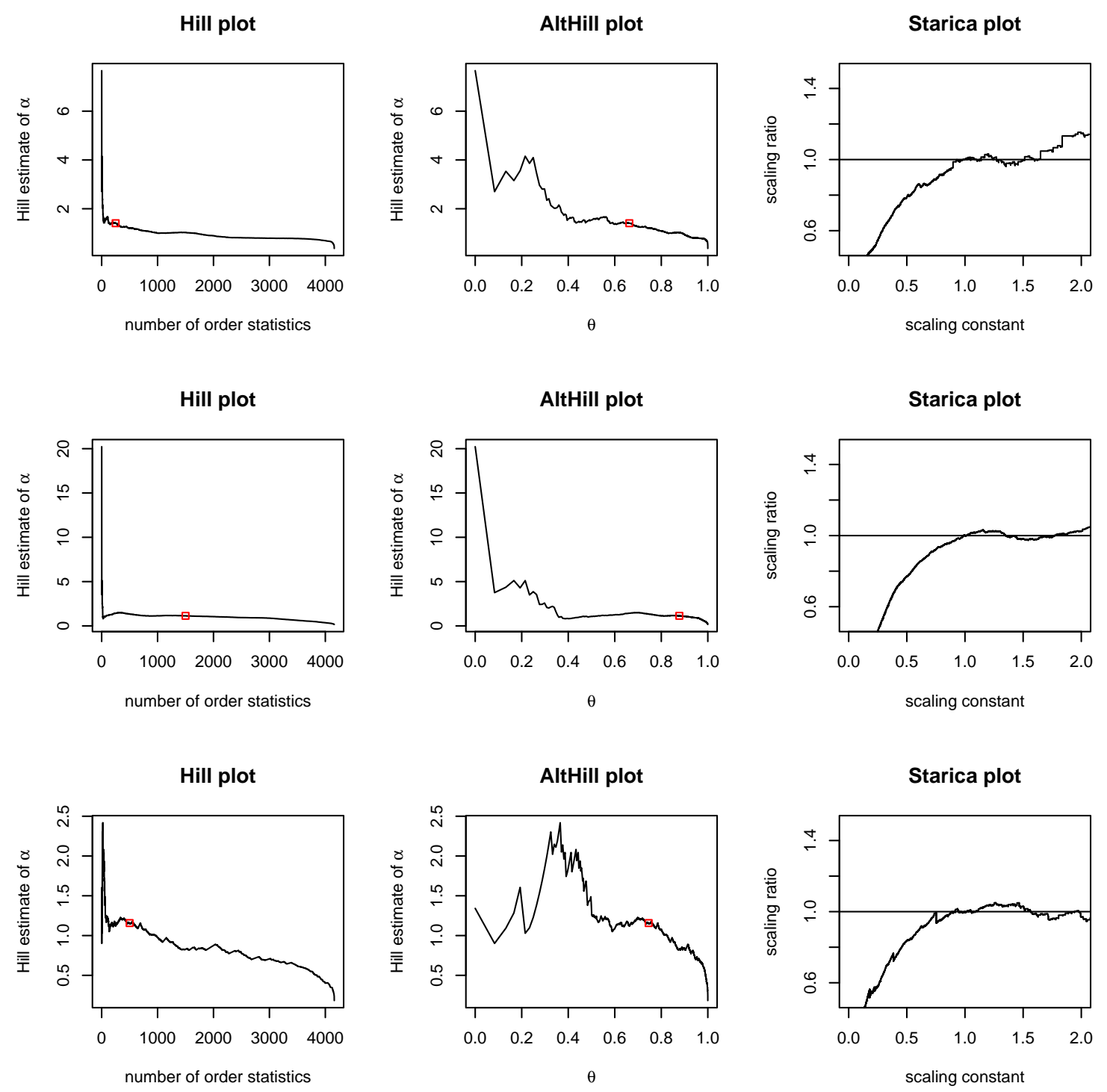

FiguRE 2. Hill plots of top) transmission length, middle) transmission rate, and bottom) transmitted file size

We end this section with some notations used later in the paper. A function $G$ is called regularly varying of index $\alpha$ and is denoted by $G \in R V_{\alpha}$ if

$$
\lim _{t \rightarrow \infty} \frac{G(x t)}{G(t)}=x^{\alpha}
$$

The left-continuous inverse of a non-decreasing right-continuous function $\Phi$ is defined to be

$$
\Phi^{\leftarrow}(y)=\inf \{x: \Phi(x) \geqslant y\} .
$$


For a random variable $X$, we denote its distribution function by $F_{X}$ and $\bar{F}_{X}(x)=1-F_{X}(x)$. The quantile function of $X$ is defined as

$$
b_{X}(T)=\inf \left\{x: \bar{F}_{X}(x) \leqslant \frac{1}{T}\right\}=\left(\frac{1}{\bar{F}_{X}}\right)^{\leftarrow}(T) .
$$

The vectors are denoted by bold letters and operations on vectors are always interpreted componentwise. We denote the vectors $(0,0)$ and $(\infty, \infty)$ by $\mathbf{0}$ and $\infty$ respectively. The two dimensional boxes are denoted by their lower left and upper right corners, for example, $(\mathbf{a}, \mathbf{b}]$ stands for $\left(a_{1}, b_{1}\right] \times\left(a_{2}, b_{2}\right]$ and we can similarly define the boxes $(\mathbf{a}, \mathbf{b}),[\mathbf{a}, \mathbf{b})$ and $[\mathbf{a}, \mathbf{b}]$.

\section{Asymptotic indePendence}

Consider iid random vectors $(X, Y),\left(X_{1}, Y_{1}\right),\left(X_{2}, Y_{2}\right), \ldots$ In extreme value theory, $X$ and $Y$ are considered asymptotically independent, if the coordinatewise sample maxima, $\left(\vee_{i=1}^{n} X_{i}, \vee_{i=1}^{n} Y_{i}\right)$, under suitable centering and scaling, converge weakly to a product measure. When both $X$ and $Y$ have regularly varying tail probabilities, this is equivalent to the existence of regularly varying functions $b_{X}$ and $b_{Y}$, such that,

$$
T \mathrm{P}\left[\left(\frac{X}{b_{X}(T)}, \frac{Y}{b_{Y}(T)}\right) \in \cdot\right] \stackrel{\mathrm{v}}{\rightarrow} \nu(\cdot) \text { on }[\mathbf{0}, \boldsymbol{\infty}] \backslash\{\mathbf{0}\},
$$

where $\nu$ is a measure satisfying $\nu((\mathbf{0}, \infty])=0$. The convergence above is vague convergence. This means that $\nu$ concentrates on the axes $\{0\} \times(0, \infty]$ and $(0, \infty] \times\{0\}$ [cf. 22, Chapter 5]. There is an equivalent formulation of the above concept where the variables are transformed so as to have the similar tails [cf. 5, Section 4], which states:

$$
T \mathrm{P}\left[\frac{\left(b_{X}^{\overleftarrow{X}}(X), b_{Y}^{\overleftarrow{Y}}(Y)\right)}{T} \in \cdot\right] \stackrel{\mathrm{v}}{\rightarrow} \tilde{\nu}(\cdot) \text { on }[\mathbf{0}, \boldsymbol{\infty}] \backslash\{\mathbf{0}\},
$$

where $\tilde{\nu}$ satisfies $\tilde{\nu}((\mathbf{0}, \infty])=0$, and $\tilde{\nu}$ is also homogeneous of index -1 . Thus if we define

$$
\Phi(\theta)=\tilde{\nu}\left\{(s, t): s \vee t>1, \frac{t}{s} \leq \tan \theta\right\}, 0 \leq \theta \leq \frac{\pi}{2}
$$

then the asymptotic independence is equivalent to the fact that $\Phi$ is supported on $\left\{0, \frac{\pi}{2}\right\}$. Motivated by the network modelling problem, we are interested in understanding how the distribution tail behavior of random variables affects tail behavior of the products of random variables. The class of distributions possessing classical asymptotic independence is too broad a class for the study of the products as is clear from the following examples.

Example 2.1. Let $U$ and $V$ be random variables with regularly varying tails of indices $-\alpha_{U}$ and $-\alpha_{V}$, with $1<\alpha_{U}, \alpha_{V}<2$. Let $b_{U}$ and $b_{V}$ be the corresponding quantile functions, defined as in (1.1). Let $B$ be a Bernoulli random variable with probability of success 0.5 , independent of $U$ and $V$. Then define

$$
(X, Y)=B(U, 0)+(1-B)(0, V) .
$$

Then we have

$$
\begin{aligned}
T \mathrm{P}\left[\left(\frac{X}{b_{U}(T)}, \frac{Y}{b_{V}(T)}\right) \in \cdot\right] & =\frac{1}{2} T \mathrm{P}\left[\left(\frac{U}{b_{U}(T)}, 0\right) \in \cdot\right]+\frac{1}{2} T \mathrm{P}\left[\left(0, \frac{V}{b_{V}(T)}\right) \in \cdot\right] \\
& \stackrel{\mathrm{v}}{\rightarrow} \frac{1}{2} \nu_{\alpha_{U}} \times \varepsilon_{0}(\cdot)+\frac{1}{2} \varepsilon_{0} \times \nu_{\alpha_{V}}(\cdot) \quad \text { on }[\mathbf{0}, \boldsymbol{\infty}] \backslash\{\mathbf{0}\},
\end{aligned}
$$


where $\varepsilon_{0}$ is the Dirac measure at 0 . Thus the limiting measure is concentrated on the axes; i. e., on the set $(\{0\} \times(0, \infty]) \cup((0, \infty] \times\{0\})$. We conclude that $(2.1)$ holds, but no interesting product behavior is possible since $X Y \equiv 0$.

In the next example, (2.1) again holds. The product $X Y$ is not degenerate, but still we cannot draw any interesting conclusion about the tail behavior of the product.

Example 2.2. Let $U, V$ and $B$ be as in the previous example. Define

$$
(X, Y)=B\left(U, U^{p}\right)+(1-B)\left(V^{p}, V\right),
$$

where $0<p \leqslant \frac{1}{2}$. Suppose $\alpha_{V}<\alpha_{U}$, so that $V$ has a heavier tail. Now observe that $\alpha_{U}<2<2 \alpha_{V}$, since $\alpha_{V}>1$, and similarly also $\alpha_{V}<2<2 \alpha_{U}$. So we have $b_{U}(T)^{p} \leqslant \sqrt{b_{U}(T)}=o\left(b_{V}(T)\right)$ and $b_{V}(T)^{p} \leqslant \sqrt{b_{V}(T)}=o\left(b_{U}(T)\right)$ as $T \rightarrow \infty$. Then

$$
\begin{aligned}
T \mathrm{P}\left[\left(\frac{X}{b_{U}(T)}, \frac{Y}{b_{V}(T)}\right) \in \cdot\right] & =\frac{T}{2} \mathrm{P}\left[\left(\frac{U}{b_{U}(T)}, \frac{U^{p}}{b_{V}(T)}\right) \in \cdot\right]+\frac{T}{2} \mathrm{P}\left[\left(\frac{V^{p}}{b_{U}(T)}, \frac{V}{b_{V}(T)}\right) \in \cdot\right] \\
& \stackrel{\mathrm{v}}{\rightarrow} \frac{1}{2} \nu_{\alpha_{U}} \times \varepsilon_{0}(\cdot)+\frac{1}{2} \varepsilon_{0} \times \nu_{\alpha_{V}}(\cdot) \quad \text { on }[\mathbf{0}, \boldsymbol{\infty}] \backslash\{\mathbf{0}\},
\end{aligned}
$$

and the limiting measure is concentrated on the axes.

Also $X Y=B U^{1+p}+(1-B) V^{1+p}$. Then, since $\alpha_{V}<\alpha_{U}$, we have,

$$
\mathrm{P}[X Y>x] \sim \frac{1}{2} \mathrm{P}\left[V^{1+p}>x\right]
$$

which is regularly varying of index $-\frac{\alpha_{V}}{1+p}$. Since $\mathrm{P}[X>\cdot] \in R V_{-\alpha_{U}}, \mathrm{P}[Y>\cdot] \in R V_{-\alpha_{V}}$, the tail behavior of $X Y$ cannot be concluded from the tail behavior of the factors even though (2.1) holds.

These examples reinforce the idea that the classical notion of asymptotic independence from extreme value theory contains little information about the tail behavior of the product. So we need to strengthen the concept.

In the following we write $\nu_{\beta}$ for the measure on $(0, \infty]$ satisfying $\nu_{\beta}((x, \infty])=x^{-\beta}, x>0, \beta>0$.

Definition 2.1. For two strictly positive random variables $X$ and $Y$, we say $Y$ is asymptotically independent of $X$, if

$$
T \mathrm{P}\left[\left(\frac{X}{b_{X}(T)}, Y\right) \in \cdot\right] \stackrel{\mathrm{v}}{\rightarrow} \nu_{\alpha_{X}} \times G(\cdot) \text { on } D:=(0, \infty] \times[0, \infty],
$$

where $G$ is a probability measure with $G((0, \infty))=1$.

The definition is not symmetric in $X$ and $Y$. Also, if $Y$ is asymptotically independent of $X$, then considering vague convergence on the set $(x, \infty] \times[0, \infty]$, we obtain, for all $x>0$,

$$
T \mathrm{P}\left[\frac{X}{b_{X}(T)}>x\right] \rightarrow x^{-\alpha_{X}}
$$

and hence $X$ has a regularly varying tail of index $-\alpha_{X}$. It should be clear that if $\bar{F}_{X} \in R V_{-\alpha}$, and $Y$ is independent of $X$, then (2.4) holds.

We consider the cone $D=(0, \infty] \times[0, \infty]$ instead of the more natural choice of $[\mathbf{0}, \infty] \backslash\{\mathbf{0}\}$ for the simple reason that a characterization of (2.4) by means of multivariate regular variation on the larger set is not possible without further assumptions. See Theorem 2.1 and Lemma 2.2.

Condition (2.4) in the definition of asymptotic independence is stronger than the usual concept of asymptotic independence in extreme value theory.

Lemma 2.1. Assume both the conditions (2.4) and (2.1) hold. Then $\nu$ satisfies $\nu((\mathbf{0}, \infty])=0$. 
Proof. Fix $x>0$. Let us define $\mathbf{x}=(x, x)$. Since $b_{X}(T) \rightarrow \infty$, we have, for all $K>0, b_{X}(T) x>K$, for sufficiently large $T$. Hence we have, for all $K>0$,

$$
\begin{aligned}
\nu((x, \infty] \times(x, \infty])=\nu((\mathbf{x}, \infty]) & =\lim _{T \rightarrow \infty} T \mathrm{P}\left[X>b_{L}(T) x, Y>b_{R}(T) x\right] \\
& \leqslant \lim _{T \rightarrow \infty} T \mathrm{P}\left[X>K, Y>b_{R}(T) x\right] \\
& =\bar{G}(K) \nu_{\alpha_{R}}(x, \infty] .
\end{aligned}
$$

Then letting $K \rightarrow \infty$, we get $\nu((\mathbf{x}, \infty])=0$, for all $x>0$.

We now characterize asymptotic independence in terms of standard multivariate regular variation on the cone $D$ [cf. 22, Chapter 5], in the spirit of the characterization of multivariate regular variation using a polar coordinate transformation [cf. 1].

Theorem 2.1. Assume $X$ and $Y$ are strictly positive, finite random variables. Suppose

$$
T \mathrm{P}\left[X>b_{X}(T)\right] \rightarrow 1
$$

Then the following are equivalent:

(i) $T \mathrm{P}\left[\left(\frac{X}{b_{X}(T)}, Y\right) \in \cdot\right] \stackrel{\mathrm{v}}{\rightarrow}\left(\nu_{\alpha} \times G\right)(\cdot)$ on $D$

for some $\alpha>0$, and $G$ a probability measure satisfying $G((0, \infty))=1$.

(ii) $T \mathrm{P}\left[\frac{(X, X Y)}{b_{X}(T)} \in \cdot\right] \stackrel{\mathrm{v}}{\rightarrow} \nu(\cdot)$ on $D$ where $\nu(\{(x, y): x>u\})>0$ for all $u>0$.

In fact, $\nu$ is homogeneous of order $-\alpha$; i.e., $\nu(u \cdot)=u^{-\alpha} \nu(\cdot)$ on $D$, and is given by

$$
\nu=\left\{\begin{array}{lll}
\left(\nu_{\alpha} \times G\right) \circ \theta^{-1} & \text { on } & (0, \infty) \times[0, \infty) \\
0 & \text { on } & D \backslash((0, \infty) \times[0, \infty))
\end{array},\right.
$$

where $\theta(x, y)=(x, x y)$, if $(x, y) \in D \backslash\{(\infty, 0)\}$ and $\theta(\infty, 0)$ is defined arbitrarily.

Remarks. The function $\theta$ as defined above is Borel-measurable, irrespective of its value at the point $(\infty, 0)$, since the singleton subset $\{(\infty, 0)\}$ is a measurable subset of $D$.

Condition (2.8) holds, for example, when $X$ has a regularly varying tail.

The measure $\nu$ as defined above is Radon. To see this, note that the relatively compact sets in $D$ are contained in $[a, \infty] \times[0, \infty]$ and

$$
\nu([a, \infty] \times[0, \infty])=\left(\nu_{\alpha} \times G\right)([a, \infty] \times[0, \infty])=a^{-\alpha}<\infty .
$$

Proof of Theorem 2.1. (i) $\Rightarrow$ (ii): Let $0<s<\infty$ and $S \in \mathcal{B}([0, \infty])$. Define

$$
V_{s, S}=\{(x, x y) \in D: s<x<\infty, y \in S\} .
$$

Now $V_{s,\left[t_{1}, t_{2}\right]}$ is relatively compact in $D$ for all $0 \leqslant t_{1} \leqslant t_{2} \leqslant \infty$. Also if $G\left(\left\{t_{1}, t_{2}\right\}\right)=0$, then we have,

$$
\begin{aligned}
T \mathrm{P}\left[\frac{(X, X Y)}{b_{X}(T)} \in V_{s,\left[t_{1}, t_{2}\right]}\right] & =T \mathrm{P}\left[\left(\frac{X}{b_{X}(T)}, Y\right) \in(s, \infty) \times\left[t_{1}, t_{2}\right]\right] \\
& \rightarrow\left(\nu_{\alpha} \times G\right)\left((s, \infty) \times\left[t_{1}, t_{2}\right]\right)=\nu\left(V_{s,\left[t_{1}, t_{2}\right]}\right),
\end{aligned}
$$

where $\nu$ is as defined in (2.9). Now, fix $s_{0} \in(0, \infty)$. Note that

$$
T \mathrm{P}\left[\frac{(X, X Y)}{b_{X}(T)} \in V_{s_{0},[0, \infty]}\right] \rightarrow \nu\left(V_{s_{0},[0, \infty]}\right)=\left(\nu_{\alpha} \times G\right)\left(\left(s_{0}, \infty\right) \times[0, \infty]\right)=s_{0}^{-\alpha},
$$


which is also strictly positive and finite. Hence $T \mathrm{P}\left[\frac{(X, X Y)}{b_{X}(T)} \in V_{s_{0},[0, \infty]}\right]$ is strictly positive and finite for all large $T$. So we can define probability measures $Q_{T}(\cdot)$ and $Q(\cdot)$ on $\left(s_{0}, \infty\right) \times[0, \infty]$ for all large $T$, by

$$
Q_{T}(\cdot)=\frac{\mathrm{P}\left[\frac{(X, X Y)}{b_{X}(T)} \in \cdot\right]}{\mathrm{P}\left[\frac{(X, X Y)}{b_{X}(T)} \in V_{s_{0},[0, \infty]}\right]} \text { and } Q(\cdot)=\frac{\nu(\cdot)}{\nu\left(V_{s_{0},[0, \infty]}\right)}
$$

Then

$$
Q_{T}\left(V_{s,\left[t_{1}, t_{2}\right]}\right) \rightarrow Q\left(V_{s,\left[t_{1}, t_{2}\right]}\right) \quad \forall s \in\left(s_{0}, \infty\right), 0 \leqslant t_{1} \leqslant t_{2} \leqslant \infty \text { with } G\left(\left\{t_{1}, t_{2}\right\}\right)=0
$$

Let

$$
\mathcal{P}=\left\{V_{s_{1},\left[t_{1}, t_{2}\right]} \backslash V_{s_{2},\left[t_{1}, t_{2}\right]}: s_{0}<s_{1}<s_{2}<\infty, 0 \leqslant t_{1} \leq t_{2} \leqslant \infty\right\} .
$$

Observe $B \in \mathcal{P}$ is a $Q$-continuity set iff $G\left(\left\{t_{1}, t_{2}\right\}\right)=0$. So by $(2.10), Q_{T}(B) \rightarrow Q(B)$ for all $Q$-continuity sets $B \in \mathcal{P}$. Also, clearly for every $\mathbf{x}$ in $\left(s_{0}, \infty\right) \times[0, \infty]$ and positive $\varepsilon$, there is an $A$ in $\mathcal{P}$, for which $\mathbf{x} \in A^{\circ} \subseteq A \subseteq B(\mathbf{x}, \varepsilon)$, where $A^{\circ}$ is the interior of $A$ and $B(\mathbf{x}, \varepsilon)$ is the ball of radius $\varepsilon$ around $\mathbf{x}$. Now $\mathcal{P}$ is a $\pi$-system. Then, by Theorem 2.3 of Billingsley [2], we have

$$
Q_{T} \Rightarrow Q \text { on }\left(s_{0}, \infty\right) \times[0, \infty] .
$$

Thus $Q_{T}(B) \rightarrow Q(B)$ for all Borel sets $B$ of $\left(s_{0}, \infty\right) \times[0, \infty]$ with boundary in $\left(s_{0}, \infty\right) \times[0, \infty]$ having zero $Q$-measure, for all $s_{0}>0$. Hence the same result holds with $Q_{T}, Q$ replaced by $T \mathrm{P}\left[\frac{(X, X Y)}{b_{X}(T)} \in \cdot\right], \nu$ respectively.

Let $K$ be relatively compact in $D$ with $\nu\left(\partial_{D} K\right)=0$. Then there exists $s_{0}>0$, such that $K \subset\left(s_{0}, \infty\right] \times[0, \infty]$. Define $B=K \cap\left(\left(s_{0}, \infty\right) \times[0, \infty]\right)$. Then $B$ is Borel in $\left(s_{0}, \infty\right) \times[0, \infty]$ and $\nu\left(\partial_{\left(s_{0}, \infty\right) \times[0, \infty]} B\right)=0$. We have, by definition of $\nu$ in $(2.9)$,

Therefore

$$
T \mathrm{P}\left[\frac{(X, X Y)}{b_{X}(T)} \in K\right]=T \mathrm{P}\left[\frac{(X, X Y)}{b_{X}(T)} \in B\right] \rightarrow \nu(B)=\nu(K) .
$$

where $\nu$ is defined as in (2.9). Thus,

$$
T \mathrm{P}\left[\frac{(X, X Y)}{b_{X}(T)} \in \cdot\right] \stackrel{\mathrm{v}}{\rightarrow} \nu(\cdot) \text { on } D
$$

$$
\nu(\{(x, y): x>u\})=\left(\nu_{\alpha} \times G\right)(\{(x, y): x>u\})=u^{-\alpha}>0, \quad \forall u>0 .
$$

(ii) $\Rightarrow$ (i): Define $U=\{(x, y) \in D: x>1\}$.

Choose integer $n_{v}$ such that $b\left(n_{v}\right) \leqslant v<b\left(n_{v}+1\right)$. Fix $0<s \leqslant \infty, 0 \leqslant t \leqslant \infty$, so that $\nu(\partial([s, \infty] \times[t, \infty]))=0$. Then

$$
\begin{aligned}
\frac{n_{v}}{n_{v}+1} \cdot \frac{\left(n_{v}+1\right) \mathrm{P}\left[\frac{(X, X Y)}{b_{X}\left(n_{v}+1\right)} \in[s, \infty] \times[t, \infty]\right]}{n_{v} \mathrm{P}\left[\frac{(X, X Y)}{b_{X}\left(n_{v}\right)} \in U\right]} & \leqslant \frac{\mathrm{P}\left[v^{-1}(X, X Y) \in[s, \infty] \times[t, \infty]\right]}{\mathrm{P}\left[v^{-1}(X, X Y) \in U\right]} \\
& \leqslant \frac{n_{v}+1}{n_{v}} \cdot \frac{n_{v} \mathrm{P}\left[\frac{(X, X Y)}{b_{X}\left(n_{v}\right)} \in[s, \infty] \times[t, \infty]\right]}{\left(n_{v}+1\right) \mathrm{P}\left[\frac{(X, X Y)}{b_{X}\left(n_{v}+1\right)} \in U\right]},
\end{aligned}
$$

and taking the limit as $v \rightarrow \infty$, we find, for $0<s \leqslant \infty$ and $0<t \leqslant \infty$, that

$$
\frac{\mathrm{P}\left[v^{-1}(X, X Y) \in[s, \infty] \times[t, \infty]\right]}{\mathrm{P}\left[v^{-1}(X, X Y) \in U\right]} \rightarrow \frac{\nu([s, \infty] \times[t, \infty])}{\nu(U)}, \quad 0<s \leqslant \infty, \quad 0 \leqslant t \leqslant \infty .
$$


Arguing as before, normalizing to probability measures and so on, we have

$$
\frac{\mathrm{P}[(X, X Y) \in v \cdot]}{\mathrm{P}[(X, X Y) \in v U]} \stackrel{\mathrm{v}}{\rightarrow} \frac{\nu(\cdot)}{\nu(U)} \text { on } D .
$$

Then, by the usual argument, [cf. 22], $\frac{\nu(\cdot)}{\nu(U)}$ is homogeneous on $D$ of order $-\alpha$, for some $\alpha>0$, and hence this is true for $\nu(\cdot)$; i. e., $\nu(s \cdot)=s^{-\alpha} \nu(\cdot)$ on $D$.

Now, by (2.8) and the fact that $X$ and $Y$ are strictly positive and finite random variables, we have,

$$
1=\lim _{T \rightarrow \infty} T \mathrm{P}\left[\frac{X}{b_{X}(T)}>1\right]=\lim _{T \rightarrow \infty} T \mathrm{P}\left[\frac{X}{b_{X}(T)}>1, Y \in(0, \infty)\right]=\nu\left(V_{1,(0, \infty)}\right) .
$$

Thus $G(\cdot):=\nu\left(V_{1, \cdot}\right)$ is a probability measure on $[0, \infty]$ with $G\left(\mathbb{R}_{+}\right)=1$.

Also, for all $s>0, S \in \mathcal{B}([0, \infty])$,

$$
\nu\left(V_{s, S}\right)=\left(\nu_{\alpha} \times G\right)((s, \infty] \times S)=\left(\nu_{\alpha} \times G\right)\left(\theta^{-1} V_{s, S}\right),
$$

and thus $\nu$ has a form as defined in (2.9).

Again, $V_{s,\left[t_{1}, t_{2}\right]}$ is a $\nu$-continuity set iff $(s, \infty] \times\left[t_{1}, t_{2}\right]$ is a $\left(\nu_{\alpha} \times G\right)$-continuity set iff $G\left(\left\{t_{1}, t_{2}\right\}\right)=0$. Thus for all $s>0$, all $0 \leqslant t_{1} \leqslant t_{2} \leqslant \infty$ with $G\left(\left\{t_{1}, t_{2}\right\}\right)=0$, i. e., $V_{s,\left[t_{1}, t_{2}\right]}$ a $\nu$-continuity set, we have, for all $s>0, S \in \mathcal{B}([0, \infty])$,

$T \mathrm{P}\left[\frac{X}{b_{X}(T)}>s, Y \in\left[t_{1}, t_{2}\right]\right]=T \mathrm{P}\left[\frac{(X, X Y)}{b_{X}(T)} \in V_{s,\left[t_{1}, t_{2}\right]}\right] \rightarrow \nu\left(V_{s,\left[t_{1}, t_{2}\right]}\right)=\left(\nu_{\alpha} \times G\right)\left((s, \infty] \times\left[t_{1}, t_{2}\right]\right)$.

Then, arguing by normalizing to probability measures as before, we get,

$$
T \mathrm{P}\left[\left(\frac{X}{b_{X}(T)}, Y\right) \in \cdot\right] \stackrel{\mathrm{v}}{\rightarrow}\left(\nu_{\alpha} \times G\right)(\cdot) \text { on } D .
$$

Remark. Observe that in the above theorem, (i) implies (ii), even without the assumption (2.8) made separately, since (i) implies $X$ has a regular varying tail and hence (2.8) holds.

Now we extend the characterization of asymptotic independence in terms of multivariate regular variation to the larger set $[\mathbf{0}, \infty] \backslash\{\mathbf{0}\}$.

Lemma 2.2. As in Theorem 2.1, assume $X$ and $Y$ are strictly positive, finite random variables satisfying (2.4). Further assume two moment conditions that $G$ has finite $\alpha$-th moment and

$$
\lim _{\varepsilon \downarrow 0} \limsup _{T \rightarrow \infty} T \mathrm{E}\left[\left(\frac{X Y}{b_{X}(T)}\right)^{\delta} \mathbf{1}_{\left[\frac{X}{b_{X}(T)} \leqslant \varepsilon\right]}\right]=0 \text { for some } \delta>0 \text {. }
$$

Then

$$
T \mathrm{P}\left[\frac{(X, X Y)}{b_{X}(T)} \in \cdot\right] \stackrel{\mathrm{v}}{\rightarrow} \tilde{\nu} \text { on }[\mathbf{0}, \infty] \backslash\{\mathbf{0}\}
$$

where

$$
\tilde{\nu}=\left\{\begin{array}{ll}
\nu & \text { on } D \\
0 & \text { on }\{0\} \times(0, \infty]
\end{array},\right.
$$

with $\nu$ defined as in (2.9). 
Remark. As defined in the lemma, $\tilde{\nu}$ is Radon on $[\mathbf{0}, \boldsymbol{\infty}] \backslash\{\mathbf{0}\}$, if $\alpha$-th moment of $G$ is finite. To see this, note that a relatively compact set in $[\mathbf{0}, \boldsymbol{\infty}] \backslash\{\mathbf{0}\}$ is contained in $[\mathbf{0}, \infty] \backslash[\mathbf{0}, \mathbf{a}]$ for some $a>0$, where $\mathbf{a}=(a, a)$. So it is enough to check the finiteness of $\tilde{\nu}([\mathbf{0}, \boldsymbol{\infty}] \backslash[\mathbf{0}, \mathbf{a}])$. We consider the set $[\mathbf{0}, \infty] \backslash[\mathbf{0}, \mathbf{a}]$ in two disjoint components, namely, $(a, \infty] \times[0, \infty]$ and $[0, a] \times(a, \infty]$. Now

$$
\tilde{\nu}((a, \infty] \times[0, \infty])=\left(\nu_{\alpha} \times G\right)((a, \infty] \times[0, \infty])=a^{-\alpha}<\infty,
$$

and

$$
\begin{aligned}
\tilde{\nu}([0, a] \times(a, \infty]) & =\nu((0, a] \times(a, \infty])=\left(\nu_{\alpha} \times G\right)\left(\left\{\mathbf{x}: 0<x_{1} \leqslant a, x_{1} x_{2}>a\right\}\right) \\
& =\int_{(1, \infty)}\left(\left(\frac{a}{x_{2}}\right)^{-\alpha}-a^{-\alpha}\right) G\left(d x_{2}\right)=a^{-\alpha} \int_{(1, \infty)} x_{2}^{\alpha} G\left(d x_{2}\right)-G((1, \infty)) .
\end{aligned}
$$

Thus $\tilde{\nu}$ is Radon iff $\int_{(1, \infty)} x_{2}^{\alpha} G\left(d x_{2}\right)<\infty$ iff $G$ has finite $\alpha$-th moment, which has been assumed.

Proof of Lemma 2.2. We have already seen in Theorem 2.1 that, (i) of Theorem 2.1 implies vague convergence in $(2.12)$ on $D$. Let $K$ be relatively compact in $[\mathbf{0}, \infty] \backslash\{\mathbf{0}\}$ with $\tilde{\nu}(\partial K)=0$. Choose $\varepsilon_{k} \downarrow 0$ such that $K_{\varepsilon_{k}}:=K \cap\left(\left[\varepsilon_{k}, \infty\right] \times[0, \infty]\right)$ satisfy $\tilde{\nu}\left(\partial K_{\varepsilon_{k}}\right)=0$. Then

$$
\liminf _{T \rightarrow \infty} T \mathrm{P}\left[\frac{(X, X Y)}{b_{X}(T)} \in K\right] \geqslant \lim _{T \rightarrow \infty} T \mathrm{P}\left[\frac{(X, X Y)}{b_{X}(T)} \in K_{\varepsilon_{k}}\right]=\nu\left(K_{\varepsilon_{k}}\right)=\tilde{\nu}\left(K_{\varepsilon_{k}}\right) .
$$

Letting $k \rightarrow \infty$, and using the definition of $\tilde{\nu}$,

$$
\liminf _{T \rightarrow \infty} T \mathrm{P}\left[\frac{(X, X Y)}{b_{X}(T)} \in K\right] \geqslant \tilde{\nu}(K \cap D)=\tilde{\nu}(K) .
$$

Since $K$ is relatively compact in $[\mathbf{0}, \infty] \backslash\{\mathbf{0}\}$, there exists $s \in(0, \infty)$ such that $K \subseteq[\mathbf{0}, \mathbf{s}]^{c}$, where $\mathbf{s}=(s, s)$. Therefore,

$$
\begin{aligned}
\limsup _{T \rightarrow \infty} T \mathrm{P}\left[\frac{(X, X Y)}{b_{X}(T)} \in K\right] \leqslant & \lim _{T \rightarrow \infty} T \mathrm{P}\left[\frac{(X, X Y)}{b_{X}(T)} \in K_{\varepsilon_{k}}\right] \\
& +\limsup _{T \rightarrow \infty} T \mathrm{P}\left[\frac{(X, X Y)}{b_{X}(T)} \in K \cap\left(\left[0, \varepsilon_{k}\right) \times(s, \infty]\right)\right] \\
\leqslant & \tilde{\nu}\left(K_{\varepsilon_{k}}\right)+\limsup _{T \rightarrow \infty} T \mathrm{P}\left[\frac{X}{b_{X}(T)}<\varepsilon_{k}, \frac{X Y}{b_{X}(T)}>s\right] \\
\leqslant & \tilde{\nu}\left(K_{\varepsilon_{k}}\right)+s^{-\delta} \limsup _{T \rightarrow \infty} T \mathrm{E}\left[\left(\frac{X Y}{b_{X}(T)}\right)^{\delta} \mathbf{1}_{\left[\frac{X}{b_{X}(T)}<\varepsilon_{k}\right]}\right] .
\end{aligned}
$$

Letting $k \rightarrow \infty$, by (ii) of Theorem 2.1 ,

$$
\limsup _{T \rightarrow \infty} T \mathrm{P}\left[\frac{(X, X Y)}{b(T)} \in K\right] \leqslant \tilde{\nu}(K) .
$$

Hence,

$$
T \mathrm{P}\left[\frac{(X, X Y)}{b(T)} \in \cdot\right] \stackrel{\mathrm{v}}{\rightarrow} \tilde{\nu}(\cdot) \text { on }[\mathbf{0}, \boldsymbol{\infty}] \backslash\{\mathbf{0}\} .
$$

In fact, it is easily seen from Theorem 2.1 and Lemma 2.2 that the converse also holds, which is summarized in the following corollary. 
Corollary 2.1. If $X$ and $Y$ are strictly positive, finite random variables with $T \mathrm{P}\left[X_{1}>b(T)\right] \rightarrow 1$. Also assume the truncated moment condition (2.11). Then $Y$ is asymptotically independent of $X$, i.e.,

$$
T \mathrm{P}\left[\left(\frac{X}{b_{X}(T)}, Y\right) \in \cdot\right] \stackrel{\mathrm{v}}{\rightarrow} \nu_{\alpha} \times G(\cdot) \text { on } D=(0, \infty] \times[0, \infty]
$$

for some $\alpha>0$, and some probability measure $G$ on $[0, \infty]$ with $G((0, \infty))=1$ and finite $\alpha$-th moment iff

$$
T \mathrm{P}\left[\frac{(X, X Y)}{b_{X}(T)} \in \cdot \stackrel{\stackrel{\mathrm{v}}{\rightarrow}}{\sim}(\cdot) \text { on }[\mathbf{0}, \infty] \backslash\{\mathbf{0}\}\right.
$$

for some Radon measure $\tilde{\nu}$ satisfying

$$
\tilde{\nu}(\{(x, y): x>u\})>0 \text { for some } u>0 .
$$

In fact, $\tilde{\nu}$ is homogeneous of order $-\alpha$ and is given as in (2.9) and (2.13).

Another equivalent formulation of asymptotic independence justifies the nomenclature and loosely says that $Y$ is asymptotically independent of $X$, if the conditional distribution of $Y$ is same as the marginal distribution, when $X$ is large.

Lemma 2.3. Assume $X$ and $Y$ are strictly positive, finite random variables. Then

$$
T \mathrm{P}\left[\left(\frac{X}{b_{X}(T)}, Y\right) \in \cdot\right] \stackrel{\mathrm{v}}{\rightarrow} \nu_{\alpha_{X}} \times G(\cdot) \text { on } D
$$

implies

$$
\mathrm{P}[Y \in \cdot \mid X>x] \Rightarrow G(\cdot) \text { as } x \rightarrow \infty,
$$

where the second convergence is the usual weak convergence. If the marginal distribution of $X$ has a regularly varying tail of index $-\alpha_{X}$, then the converse also holds.

Proof. Observe that (2.15) holds iff for all $x>0$ and $y \geqslant 0$, we have

$$
T \mathrm{P}\left[\frac{X}{b_{X}(T)}>x, Y \leqslant y\right] \rightarrow x^{-\alpha_{X}} G(y) .
$$

However,

$$
T \mathrm{P}\left[\frac{X}{b_{X}(T)}>x, Y \leqslant y\right]=T \mathrm{P}\left[\frac{X}{b_{X}(T)}>x\right] \mathrm{P}\left[Y \leqslant y \mid X>b_{X}(T) x\right],
$$

and, by the assumption about the nature of the marginal distribution of $X$ or by asymptotic independence, we have,

Thus (2.17) holds iff

$$
T \mathrm{P}\left[\frac{X}{b_{X}(T)}>x\right] \rightarrow x^{-\alpha_{L}}
$$

$$
\mathrm{P}\left[Y \leqslant y \mid X>b_{X}(T) x\right] \rightarrow G(y)
$$

as $T \rightarrow \infty$, for all $y \geqslant 0$. This is equivalent to $(2.16)$, since $b_{X}(T) \rightarrow \infty$.

The required truncated moment condition (2.11) holds, when $X$ and $Y$ are independent random variables with $X$ having regularly varying tails of index $-\alpha_{X}$ and $Y$ having all moments less than $\alpha_{Y}$ finite, where $\alpha_{X}<\alpha_{Y}$.

Lemma 2.4. Let $X$ and $Y$ be independent random variables taking values in $(0, \infty)$ with respective marginal distributions $F_{X}$ and $F_{Y}$ and quantile functions $b_{X}$ and $b_{Y}$. Let the index of regular variation of $\bar{F}_{X}$ be $-\alpha_{X}$ and $\mathrm{E}\left[Y^{\delta}\right]<\infty$ for $\delta<\alpha_{Y}$ with $\alpha_{X}<\alpha_{Y}$. Then (2.11) holds for $\delta \in\left(\alpha_{X}, \alpha_{Y}\right)$. 
Proof. We have by independence and $\delta<\alpha_{Y}$ and Karamata's theorem that as $T \rightarrow \infty$,

$$
\begin{aligned}
T \mathrm{E} & {\left[\left(\frac{X}{b_{X}(T)} Y\right)^{\delta} \mathbf{1}_{\left[\frac{X}{b_{X}(T)} \leqslant \varepsilon\right]}\right]=\mathrm{E}\left(Y^{\delta}\right) \frac{T}{\left(b_{X}(T)\right)^{\delta}} \mathrm{E}\left[X^{\delta} \mathbf{1}_{\left[\frac{X}{b_{X}(T)} \leqslant \varepsilon\right]}\right] } \\
& \sim \frac{\alpha_{X}}{\delta-\alpha_{X}} \mathrm{E}\left(Y^{\delta}\right) \varepsilon^{\delta} T \bar{F}_{L}\left(b_{X}(T) \varepsilon\right) \sim \frac{\alpha_{X}}{\delta-\alpha_{X}} \mathrm{E}\left(Y^{\delta}\right) \varepsilon^{\delta-\alpha_{X}},
\end{aligned}
$$

which goes to 0 as $\varepsilon \rightarrow 0$, since $\delta>\alpha_{X}$. Thus, for all $\delta \in\left(\alpha_{X}, \alpha_{Y}\right)$,

$$
\lim _{\varepsilon \downarrow 0} \limsup _{T \rightarrow \infty} \mathrm{E}\left[\left(\frac{X}{b_{X}(T)} Y\right)^{\delta} \mathbf{1}_{\left[\frac{X}{b_{X^{(T)}}}<\varepsilon\right]}\right]=0 .
$$

\section{Products And Asymptotic independence}

We are now ready to study the product of two asymptotically independent random variables.

Theorem 3.1. Suppose $X$ and $Y$ are two strictly positive, finite random variables, which are asymptotically independent. Assume the moment conditions that (2.4) holds with the limit distribution function $\nu_{\alpha_{X}} \times G$, and $G$ has $\alpha_{X}$-th moment finite and (2.11) holds. Then

$$
T \mathrm{P}\left[\frac{X Y}{b_{X}(T)}>z\right] \sim z^{-\alpha_{X}} \int_{0}^{\infty} u^{\alpha_{X}} G(d u),
$$

and hence the quantile function of $Z=X Y$ satisfies

$$
b_{Z}(T) \sim\left(\int_{0}^{\infty} u^{\alpha_{X}} G(d u)\right)^{\frac{1}{\alpha_{X}}} b_{X}(T) .
$$

Proof. Let

$$
A_{\varepsilon}=\left\{(x, y): \varepsilon<x<\varepsilon^{-1}, x y>z\right\} .
$$

Note $A_{\varepsilon}$ is relatively compact in $D$ and

$$
\partial A_{\varepsilon}=\left(\{\varepsilon\} \times\left[\frac{z}{\varepsilon}, \infty\right]\right) \cup\left(\left\{\varepsilon^{-1}\right\} \times[z \varepsilon, \infty]\right) \cup\left\{(x, y): \varepsilon<x<\varepsilon^{-1}, x y=z\right\} .
$$

Choose a sequence $\varepsilon_{k} \downarrow 0$ such that $\left(\nu_{\alpha_{L}} \times G\right)\left(\partial A_{\varepsilon_{k}}\right)=0$, for all k. Then

$$
\liminf _{T \rightarrow \infty} T \mathrm{P}\left[\frac{X}{b_{X}(T)} Y>z\right] \geqslant \lim _{T \rightarrow \infty} T \mathrm{P}\left[\left(\frac{X}{b_{X}(n)}, Y\right) \in A_{\varepsilon_{k}}\right]=\left(\nu_{\alpha_{L}} \times G\right)\left(A_{\varepsilon_{k}}\right)
$$

by (IB). Taking the limit as $k \rightarrow \infty$,

$$
\begin{aligned}
\liminf _{T \rightarrow \infty} T \mathrm{P}\left[\frac{X}{b_{X}(T)} Y>z\right] & \geqslant\left(\nu_{\alpha_{L}} \times G\right)(\{(x, y): x y>z, 0<x<\infty\}) \\
& =\left(\nu_{\alpha_{L}} \times G\right)(\{(x, y): x y>z, 0<x \leq \infty\})
\end{aligned}
$$

since $\nu_{\alpha_{L}}(\{\infty\})=0$. Also, since $\left\{(x, y) \in D: x \geq \varepsilon_{k}^{-1}\right\}$ is a $\nu_{\alpha_{L}} \times G$ continuity set, we have,

$$
\begin{aligned}
\limsup _{T \rightarrow \infty} T \mathrm{P}\left[\frac{X}{b_{X}(T)} Y>z\right] \leqslant & \lim _{T \rightarrow \infty} T \mathrm{P}\left[\left(\frac{X}{b_{X}(T)}, Y\right) \in A_{\varepsilon_{k}}\right]+\lim _{T \rightarrow \infty} T \mathrm{P}\left[\frac{X}{b_{X}(T)} \geqslant \varepsilon_{k}^{-1}\right] \\
& +\limsup _{T \rightarrow \infty} T \mathrm{P}\left[\frac{X}{b_{X}(T)} Y>z, \frac{X}{b_{X}(T)} \leqslant \varepsilon_{k}\right]
\end{aligned}
$$




$$
\begin{aligned}
= & \left(\nu_{\alpha_{X}} \times G\right)\left(A_{\varepsilon_{k}}\right)+\nu_{\alpha_{X}}\left(\left[\varepsilon_{k}^{-1}, \infty\right]\right) \\
& +\limsup _{T \rightarrow \infty} T \mathrm{P}\left[\left(\frac{X}{b_{X}(T)} Y\right) \mathbf{1}_{\left[\frac{X}{b_{X}(T)} \leqslant \varepsilon_{k}\right]}>z\right] \\
\leqslant & \left.\left(\nu_{\alpha_{X}} \times G\right)\left(A_{\varepsilon_{k}}\right)+\varepsilon_{k}^{\alpha_{X}}+z^{-\delta} \limsup _{T \rightarrow \infty} T \mathrm{E}\left[\left(\frac{X}{b_{X}(T)} Y\right)^{\delta} \mathbf{1}_{\left[\frac{X}{b_{X^{(T)}}} \leqslant \varepsilon_{k}\right.}\right]\right] .
\end{aligned}
$$

Taking limits as $k \rightarrow \infty$, and using (2.11) and the fact $\alpha_{L}>0$

$$
\begin{aligned}
\limsup _{T \rightarrow \infty} T \mathrm{P}\left[\frac{X}{b_{X}(T)} Y>z\right] & \leqslant\left(\nu_{\alpha_{L}} \times G\right)(\{(x, y): x y>z, 0<x<\infty\}) \\
& =\left(\nu_{\alpha_{L}} \times G\right)(\{(x, y) \in D: x y>z\} .
\end{aligned}
$$

Thus,

$$
\begin{aligned}
\limsup _{T \rightarrow \infty} T \mathrm{P}\left[\frac{X}{b_{X}(T)} Y>z\right] \leqslant & \left(\nu_{\alpha_{Y}} \times G\right)(\{(x, y) \in D: x y>z\} \\
& =\int_{0}^{\infty} \nu_{\alpha_{X}}\left(\left(\frac{z}{u}, \infty\right]\right) G(d u)=z^{-\alpha_{X}} \int_{0}^{\infty} u^{\alpha_{X}} G(d u) .
\end{aligned}
$$

Therefore

$$
\lim _{T \rightarrow \infty} T \mathrm{P}\left[\frac{X}{b_{X}(T)} Y\left(\int_{0}^{\infty} u^{\alpha_{X}} G(d u)\right)^{-\frac{1}{\alpha_{L}}}>z\right]=z^{-\alpha_{X}}
$$

and hence

$$
b_{Z}(T) \sim\left(\int_{0}^{\infty} u^{\alpha_{X}} G(d u)\right)^{\frac{1}{\alpha_{X}}} b_{X}(T) \sim b_{X}\left(\int_{0}^{\infty} u^{\alpha_{X}} G(d u) T\right) .
$$

Thus, under further moment conditions, the product of two asymptotically independent random variables with regularly varying tails again has a regularly varying tail, whose behavior is similar to that of the heavier one of the two factors. This is a generalization of Breiman [3]'s result about the product of two independent random variables.

The product of two random variables, which are not asymptotically independent, but whose tails satisfy multivariate regular variation, offers contrasting behavior to the asymptotically independent case.

Proposition 3.1. Suppose $(X, Y)$ is multivariate regularly varying in the sense that there exists regularly varying functions $b_{X}, b_{Y}$, such that

$$
T \mathrm{P}\left[\left(\frac{X}{b_{X}(T)}, \frac{Y}{b_{Y}(T)}\right) \in \cdot\right] \stackrel{\mathrm{v}}{\rightarrow} \nu(\cdot) \not \equiv 0
$$

on $[\mathbf{0}, \infty] \backslash\{\mathbf{0}\}$ and $\nu((\{\infty\} \times(0, \infty]) \cup((0, \infty] \times\{\infty\}))=0$ and $\nu((\mathbf{0}, \infty])>0$.

Then for some $\alpha_{X}>0, \alpha_{Y}>0$, we have

$$
\begin{aligned}
& \mathrm{P}[X>\cdot] \in R V_{-\alpha_{X}}, \\
& \mathrm{P}[Y>\cdot] \in R V_{-\alpha_{Y}}
\end{aligned}
$$


and

$$
\mathrm{P}[X Y>\cdot] \in R V_{-\frac{\alpha_{X} \alpha_{Y}}{\alpha_{X}+\alpha_{Y}}}
$$

Proof. Let $b_{X}$ and $b_{Y}$ be regularly varying with indices $1 / \alpha_{X}$ and $1 / \alpha_{Y}$ respectively. Now, for any $x>0$, such that $(x, \infty] \times[0, \infty]$ is a $\nu$-continuity set, we have,

$$
T \mathrm{P}\left[\frac{X}{b_{X}(T)}>x\right]=T \mathrm{P}\left[\left(\frac{X}{b_{X}(T)}, \frac{Y}{b_{Y}(T)}\right) \in(x, \infty] \times[0, \infty]\right] \rightarrow \nu((x, \infty] \times[0, \infty])=: K_{x},
$$

where $K_{x}$ is positive and finite for some $x_{0}>0$, since $\nu((\mathbf{0}, \infty])>0$.

Now, we have, for any $x>0$,

$$
\begin{aligned}
T \mathrm{P}\left[\frac{X}{b_{X}(T)}>x\right] & =T \mathrm{P}\left[\frac{X}{\frac{x}{x_{0}} b_{X}(T)}>x_{0}\right] \\
& \sim\left(\frac{x}{x_{0}}\right)^{-\alpha}\left(\frac{x}{x_{0}}\right)^{\alpha} T \mathrm{P}\left[\frac{X}{b_{X}\left(\left(\frac{x}{x_{0}}\right)^{\alpha} T\right)}>x_{0}\right] \\
& \rightarrow\left(x_{0}\right)^{\alpha} K_{x_{0}} x^{-\alpha}
\end{aligned}
$$

and hence $\mathrm{P}[X>\cdot] \in R V_{-\alpha_{X}}$. Similarly, we can check that $\mathrm{P}[Y>\cdot] \in R V_{-\alpha_{Y}}$.

Define for $z>0$ and any positive number $K$,

$$
A_{K, z}:=\{(x, y): x y>z, x \leqslant K, y \leqslant K\} .
$$

Then, for any $z>0$, we have,

$$
T \mathrm{P}\left[\frac{X Y}{b_{X}(T) b_{Y}(T)}>z\right] \geqslant T \mathrm{P}\left[\left(\frac{X}{b_{X}(T)}, \frac{Y}{b_{Y}(T)}\right) \in A_{K, z}\right] .
$$

Then, letting $T$ got to $\infty$ first, and then letting $K$ go to $\infty$ through a sequence so that $A_{K, z}$ is a $\nu$-continuity set, we have

$$
\limsup _{T \rightarrow \infty} T \mathrm{P}\left[\frac{X Y}{b_{X}(T) b_{Y}(T)}>z\right] \geqslant \nu(\{(x, y): x y>z\}) .
$$

On the other hand, we have,

$$
\begin{aligned}
T \mathrm{P}\left[\frac{X Y}{b_{X}(T) b_{Y}(T)}>z\right] \leqslant & T \mathrm{P}\left[\left(\frac{X}{b_{X}(T)}, \frac{Y}{b_{Y}(T)}\right) \in A_{K, z}\right] \\
& +T \mathrm{P}\left[\frac{X}{b_{X}(T)}>K\right]+T \mathrm{P}\left[\frac{X}{b_{X}(T)}>K\right]
\end{aligned}
$$

Now, by regularly varying tails of $X$ and $Y$, the last two terms converge to $K^{-\alpha_{X}}$ and $K^{-\alpha_{Y}}$ respectively, as $T \rightarrow \infty$. Then letting $K$ go to $\infty$ through a sequence so that $A_{K, z}$ is a $\nu$-continuity set, they go to zero. Hence, we have

$$
\liminf _{T \rightarrow \infty} T \mathrm{P}\left[\frac{X Y}{b_{X}(T) b_{Y}(T)}>z\right] \leqslant \nu(\{(x, y): x y>z\}) .
$$

Thus,

$$
T \mathrm{P}\left[\frac{X Y}{b_{X}(T) b_{Y}(T)}>z\right] \rightarrow \nu(\{(x, y): x y>z\})
$$


Then, since $b_{X} b_{Y}$ is a regularly varying function of index $\frac{\alpha_{X}+\alpha_{Y}}{\alpha_{X} \alpha_{Y}}$, and $\nu(\{(x, y): x y>z\})>0$ for some $z>0$, we have, arguing as in the case of $X$,

$$
\mathrm{P}[X Y>\cdot] \in R V_{-\frac{\alpha_{X} \alpha_{Y}}{\alpha_{X}+\alpha_{Y}}} \cdot
$$

In Proposition 3.1, if $\alpha_{X}$ and $\alpha_{Y}$ are between 1 and 2, i.e., $X$ and $Y$ have finite mean but infinite variance, then the product $X Y$ has a regularly varying tail of index $-\frac{\alpha_{X} \alpha_{Y}}{\alpha_{X}+\alpha_{Y}} \in\left(\frac{1}{2}, 1\right)$, i.e., the product has a much heavier tail with infinite mean. This result contrasts with Theorem 3.1, where the product of asymptotically independent random variables has tail behavior similar to the factor with the heavier tail.

The following example shows that asymptotic independence as defined here is not enough to conclude something meaningful about the product, and the truncated moment condition (2.11) cannot be dropped. Condition (2.4) holds, but condition (2.11) fails and we are unable to conclude anything meaningful about the tail behavior of the product using the tail behavior of the factors.

Example 3.1. Suppose we have independent vectors $\left(U_{1}, V_{1}\right),\left(U_{2}, V_{2}\right)$ which are independent of the Bernoulli random variable $B$ with probability of success 0.5. We assume

(i) The random variables $\left(U_{1}, V_{1}\right)$ are independent with

$$
\mathrm{P}\left[U_{1}>\cdot\right] \in R V_{-\alpha_{1}}, \mathrm{P}\left[V_{1}>\cdot\right] \in R V_{-\alpha_{2}}
$$

with

$$
1<\alpha_{2}<\alpha_{1}<2
$$

so that $V_{1}$ has the heavier tail. Let $b_{1}$ and $b_{2}$ be quantile functions of $U_{2}$ and $V_{2}$ respectively.

(ii) The random variables $\left(U_{2}, V_{2}\right)$ are dependent with multivariate regularly varying distribution in the sense that there exists regularly varying functions $b_{3}$ and $b_{4}$ of indices $1 / \alpha_{3}$ and $1 / \alpha_{4}$ respectively, such that

$$
T \mathrm{P}\left[\left(\frac{U_{2}}{b_{3}(T)}, \frac{V_{2}}{b_{4}(T)}\right) \in \cdot \stackrel{\mathrm{v}}{\rightarrow} \nu^{*}(\cdot)\right.
$$

on $[\mathbf{0}, \infty] \backslash\{\mathbf{0}\}$, where $\nu^{*}((\mathbf{0}, \infty])>0$ but $\nu^{*}(\{\infty\} \times(0, \infty] \cup(0, \infty] \times\{\infty\})=0$ and $1<\alpha_{4}<\alpha_{3}<2$. Then by Proposition 3.1,

$$
\mathrm{P}\left[U_{2}>\cdot\right] \in R V_{-\alpha_{3}} \text { and } \mathrm{P}\left[V_{2}>\cdot\right] \in R V_{-\alpha_{4}},
$$

and $V_{2}$ has a heavier tail.

(iii) Assume further that

$$
\alpha_{1}<\alpha_{3}, \alpha_{2}<\alpha_{4}
$$

and define

$$
(X, Y)=B\left(U_{2}, V_{2}\right)+(1-B)\left(U_{1}, V_{1}\right)
$$

We have the following conclusions.

(1) We have

$$
\begin{gathered}
P[X>x]=\frac{1}{2} P\left[U_{1}>x\right]+\frac{1}{2} P\left[U_{2}>x\right] \in R V_{-\alpha_{1}}, \\
P[Y>x]=\frac{1}{2} P\left[V_{1}>x\right]+\frac{1}{2} P\left[V_{2}>x\right] \in R V_{-\alpha_{2}}
\end{gathered}
$$

so that $Y$ has the heavier tail. 
(2) Define the measure $\nu_{0}=\frac{1}{2} \varepsilon_{0} \times \nu_{\alpha_{2}}+\frac{1}{2} \nu_{\alpha_{1}} \times \varepsilon_{0}$, and then on $[\mathbf{0}, \infty] \backslash\{\mathbf{0}\}$

$$
T \mathrm{P}\left[\left(\frac{X}{b_{1}(T)}, \frac{Y}{b_{2}(T)}\right) \in \cdot\right] \stackrel{\mathrm{v}}{\rightarrow} \nu_{0} \text {. }
$$

To see this, note

$$
T \mathrm{P}\left[\left(\frac{X}{b_{1}(T)}, \frac{Y}{b_{2}(T)}\right) \in \cdot\right]=\frac{T}{2} \mathrm{P}\left[\left(\frac{U_{1}}{b_{1}(T)}, \frac{V_{1}}{b_{2}(T)}\right) \in \cdot\right]+\frac{T}{2} \mathrm{P}\left[\left(\frac{U_{2}}{b_{1}(T)}, \frac{V_{2}}{b_{2}(T)}\right) \in \cdot\right] \text {, }
$$

and the second term goes to zero since $b_{i} \in R V_{\alpha_{i}-1}, \quad i=1, \ldots, 4$, and (3.2) imply

$$
b_{3}(T)=o\left(b_{1}(T)\right), \quad b_{4}(T)=o\left(b_{2}(T)\right)
$$

and the vague limit of the first term is $\nu_{0}$ on $[\mathbf{0}, \boldsymbol{\infty}] \backslash\{\mathbf{0}\}$.

(3) We obtain a different vague limit on the cone $(\mathbf{0}, \infty]$. For $x>0, y>0$, we have, using assumption (ii), that

$$
\begin{aligned}
T \mathrm{P}\left[\left(\frac{X}{b_{3}(T)}, \frac{Y}{b_{4}(T)}\right) \in(x, \infty] \times(y, \infty]\right]= & \frac{1}{2} T \mathrm{P}\left[\frac{U_{2}}{b_{3}(T)}>x, \frac{V_{2}}{b_{4}(T)}>y\right] \\
& +\frac{1}{2} T \mathrm{P}\left[\frac{U_{1}}{b_{3}(T)}>x\right] \mathrm{P}\left[\frac{V_{1}}{b_{4}(T)}>y\right] \\
\rightarrow & \frac{1}{2} \nu^{*}((x, \infty] \times(y, \infty])+0
\end{aligned}
$$

and thus the vague limit on $(\mathbf{0}, \infty]$ is $\frac{1}{2} \nu^{*}$. To verify the limit of 0 for the second term, note $b_{i}^{\leftarrow} \in R V_{\alpha_{i}}, i=1, \ldots, 4$ and as $T \rightarrow \infty$

$$
\begin{aligned}
& T P\left[U_{1}>b_{3}(T) x\right] P\left[V_{1}>b_{4}(T) y\right] \sim \frac{T}{b_{1}^{\leftarrow}\left(b_{3}(T) x\right) b_{2}^{\leftarrow}\left(b_{4}(T) y\right)} \\
& \sim \frac{T x^{-\alpha_{1}} y^{-\alpha_{2}}}{b_{1}^{\leftarrow} \circ b_{3}(T) b_{2}^{\leftarrow} \circ b_{4}(T)}
\end{aligned}
$$

which as a function of $T$ is regularly varying with index $1-\frac{\alpha_{1}}{\alpha_{3}}-\frac{\alpha_{2}}{\alpha_{4}}$. The result follows if we show $\frac{\alpha_{1}}{\alpha_{3}}+\frac{\alpha_{2}}{\alpha_{4}}>1$. However

$$
\frac{\alpha_{1}}{\alpha_{3}}+\frac{\alpha_{2}}{\alpha_{4}}>\frac{1}{2}\left(\alpha_{1}+\alpha_{2}\right)>\frac{2}{2}=1
$$

since $1<\alpha_{i}<2$, for $i=1, \ldots, 4$.

(4) We have that

$$
\mathrm{P}[X Y>\cdot] \in R V_{-\frac{\alpha_{3} \alpha_{4}}{\alpha_{3}+\alpha_{4}}} \text {, and } \frac{1}{2}<\frac{\alpha_{3} \alpha_{4}}{\alpha_{3}+\alpha_{4}}<1
$$

Note

$$
X Y=B U_{2} V_{2}+(1-B) U_{1} V_{1}
$$

so that

$$
\mathrm{P}[X Y>\cdot]=\frac{1}{2} \mathrm{P}\left[U_{2} V_{2}>\cdot\right]+\frac{1}{2} \mathrm{P}\left[U_{1} V_{1}>\cdot\right] .
$$

From Breiman [3] or Lemma 2.4 and Theorem 3.1, we have $\mathrm{P}\left[U_{1} V_{1}>\cdot\right] \in R V_{-\alpha_{2}}$, and from Proposition 3.1, $\mathrm{P}\left[U_{1} V_{1}>\cdot\right] \in R V_{-\frac{\alpha_{3} \alpha_{4}}{\alpha_{3}+\alpha_{4}}}$. But

$$
\frac{\alpha_{3} \alpha_{4}}{\alpha_{3}+\alpha_{4}}<1<\alpha_{2}
$$


and thus

$$
\mathrm{P}[X Y>\cdot] \sim \frac{1}{2} \mathrm{P}\left[U_{2} V_{2}>\cdot\right]
$$

which is surprisingly heavy in view of the fact that

$$
\mathrm{P}[X>\cdot] \in R V_{-\alpha_{1}} \text { and } \mathrm{P}[Y>\cdot] \in R V_{-\alpha_{2}},
$$

and $1<\alpha_{1}, \alpha_{2}<2$.

We conclude that the tail of the product is hidden from a condition like (2.1) or knowledge of the marginal distributions.

(5) We have $Y$ is asymptotically independent of $X$, but the truncated moment condition (2.11) fails. For the asymptotic independence condition (2.4), we have,

$$
T \mathrm{P}\left[\frac{X}{b_{1}(T)}>x, Y \leqslant y\right]=\frac{T}{2} \mathrm{P}\left[\frac{U_{1}}{b_{1}(T)}>x, V_{1} \leqslant y\right]+\frac{T}{2} \mathrm{P}\left[\frac{U_{2}}{b_{1}(T)}>x\right] \mathrm{P}\left[V_{2} \leqslant y\right] .
$$

Then the first term converges to $\left(\frac{1}{2} \nu_{\alpha_{1}} \times F_{V_{1}}\right)((x, \infty] \times[0, y])$, where $F_{V_{1}}$ denotes the distribution function of $V_{1}$. Also,

$$
\frac{T}{2} \mathrm{P}\left[\frac{U_{2}}{b_{1}(T)}>x, V_{2} \leqslant y\right] \leqslant \frac{T}{2} \mathrm{P}\left[\frac{U_{2}}{b_{3}(T)}>\frac{b_{1}(T)}{b_{3}(T)} x\right] \rightarrow 0 .
$$

since $b_{3}(T)=o\left(b_{1}(T)\right)$. Thus if we define $b_{X}(T)=2^{-1 / \alpha_{1}} b_{1}(T)$, then

$$
T \mathrm{P}\left[\left(\frac{X}{b_{X}(T)}, Y\right) \in \cdot\right] \stackrel{\mathrm{v}}{\rightarrow}\left(\nu_{\alpha_{1}} \times F_{V_{1}}\right)(\cdot)
$$

on $D$.

For the truncated moment condition (2.11), observe that,

$$
\begin{aligned}
T \mathrm{E}\left[\left(\frac{X}{b_{1}(T)} Y\right)^{\delta} \mathbf{1}_{\left.\left[\frac{X}{b_{1}(T)}<\varepsilon\right]\right]=}\right. & \frac{T}{2} \mathrm{E}\left[\left(\frac{U_{1}}{b_{1}(T)}\right)^{\delta} \mathbf{1}_{\left[\frac{U_{1}}{b_{1}(T)}<\varepsilon\right]}\right] \mathrm{E}\left[V_{1}^{\delta}\right] \\
& +\frac{T}{2} \mathrm{E}\left[\left(\frac{U_{2}}{b_{1}(T)} V_{2}\right)^{\delta} \mathbf{1}_{\left[\frac{U_{2}}{b_{1}(T)}<\varepsilon\right]}\right]
\end{aligned}
$$

So, if $\delta \geqslant \alpha_{2}$, then $\mathrm{E}\left(V_{1}^{\delta}\right)=\infty$ and hence condition (2.11) fails. Also a closer look at the proof of Lemma 2.4 will show

$$
\lim _{\varepsilon \downarrow 0} \limsup _{T \rightarrow \infty} T \mathrm{E}\left[\left(\frac{X}{b_{1}(T)}\right)^{\delta} \mathbf{1}_{\left[\frac{U_{1}}{b_{1}(T)}<\varepsilon\right]}\right]=0
$$

iff $\delta>\alpha_{1}>\alpha_{2}$. Thus, for no positive $\delta$, the first term in the right side of (3.3) can go to zero. Since $\frac{b_{X}(T)}{b_{1}(T)}=2^{-1 / \alpha_{1}}$, condition (2.11) fails even if we scale by $b_{X}$.

We can also similarly check that $X$ is asymptotically independent of $Y$ with $b_{Y}=$ $2^{-1 / \alpha_{2}} b_{2}$, and the limiting distribution $\nu_{\alpha_{2}} \times F_{U_{1}}$. However, the truncated moment condition (2.11) still fails for the pair $(Y, X)$.

So we observe that the asymptotic independence condition (2.4) is insufficient to conclude something about the tail behavior of the product and the truncated moment condition (2.11) is indispensable. 


\section{The infinite source Poisson model}

We use the concept of asymptotic independence to study a network traffic model with random transmission rate. Consider the $\mathrm{M} / \mathrm{G} / \infty$ input model of incoming traffic to a communication network. Let $\left\{\Gamma_{k}, k \geq 1\right\}$ denote the points of a homogeneous Poisson process on $[0, \infty)$ with rate $\lambda$. Suppose at time $\Gamma_{k}$, a source starts a transmission, and continues to transmit for a period of length $L_{k}$, at a fixed rate $R_{k}$, both chosen at random. The total volume of traffic injected into the network between 0 and $t$ is

$$
A(t)=\sum_{k=1}^{\infty}\left(\left(t-\Gamma_{k}\right)+\wedge L_{k}\right) R_{k}, t \geq 0 .
$$

We assume that $\left(L_{k}, R_{k}\right)$ are iid with joint distribution function $F$ and let $F_{L}$ and $F_{R}$ be the marginal distributions of $L_{k}$ and $R_{k}$ respectively. We make the following assumptions on the distribution of $\left(L_{k}, R_{k}\right)$ :

$$
\begin{gathered}
F\left(\mathbb{R}_{+}^{2}\right)=1, \text { where } \mathbb{R}_{+}^{2}=(\mathbf{0}, \infty), \\
\bar{F}_{L}(x)=\in R V_{-\alpha_{L}} \text { and } \bar{F}_{R}(x) \in R V_{-\alpha_{R}}, \alpha_{L}, \alpha_{R} \in(1,2) .
\end{gathered}
$$

We denote the quantile functions of $L_{1}$ and $R_{1}$ by $b_{L}$ and $b_{R}$ respectively. Also, let $b_{P}$ denote the quantile function of the product $L_{1} R_{1}$, namely,

$$
b_{P}(T)=\inf \left\{z: \mathrm{P}\left[L_{1} R_{1}>z\right] \leqslant T^{-1}\right\} .
$$

We make further assumptions on the joint distribution $F$ to analyze the total traffic process. We have two sets of assumptions depending on which of the two variables, the transmission length and the transmission rate, has heavier tail.

Case I: The transmission length has a heavier tail.

(IA) $\alpha_{L}<\alpha_{R}$.

(IB) $R_{1}$ is asymptotically independent of $L_{1}$, namely

$$
T \mathrm{P}\left[\left(\frac{L_{1}}{b_{L}(T)}, R_{1}\right) \in \cdot\right] \stackrel{\mathrm{v}}{\rightarrow} \nu_{\alpha_{L}} \times G(\cdot) \text { on } D:=(0, \infty] \times[0, \infty],
$$

where $G$ is a probability measure with $G\left(\mathbb{R}_{+}\right)=1$ with finite $\alpha_{L}$-th moment.

(IC) $\left(L_{1}, R_{1}\right)$ satisfy the truncated moment condition (2.11), namely,

$$
\lim _{\varepsilon \downarrow 0} \limsup _{T \rightarrow \infty} T \mathrm{E}\left[\left(\frac{L_{1}}{b_{L}(T)} R_{1}\right)^{\delta} \mathbf{1}_{\left[\frac{L_{1}}{b_{L}(T)}<\varepsilon\right]}\right]=0 \text { for some } \delta>0 .
$$

Case II: The transmission rate has a heavier tail.

(IIA) $\alpha_{R}<\alpha_{L}$.

(IIB) $L_{1}$ is asymptotically independent of $R_{1}$, namely

$$
T \mathrm{P}\left[\left(\frac{R_{1}}{b_{R}(T)}, L_{1}\right) \in \cdot\right] \stackrel{\mathrm{v}}{\rightarrow} \nu_{\alpha_{R}} \times G(\cdot) \text { on } D:=(0, \infty] \times[0, \infty],
$$

where $G$ is a probability measure with $G\left(\mathbb{R}_{+}\right)=1$ with finite $\alpha_{R^{-}}$th moment.

(IIC) $\left(R_{1}, L_{1}\right)$ satisfy the truncated moment condition (2.11), namely,

$$
\lim _{\varepsilon \downarrow 0} \limsup _{T \rightarrow \infty} T E\left[\left(\frac{R_{1}}{b_{R}(T)} L_{1}\right)^{\delta} \mathbf{1}_{\left[\frac{R_{1}}{b_{R}(T)}<\varepsilon\right]}\right]=0 \text { for some } \delta>0 \text {. }
$$


We need to distinguish between the above two cases I and II, since the object of our analysis, the total traffic process, $\{A(t): t \geqslant 0\}$ is not symmetric in the transmission rate and the transmission length. In light of Theorem 2.1, we can rewrite the conditions (IB) and (IIB) in terms of multivariate regular variation on the cone $D$ :

$\left(\mathrm{IB}^{\prime}\right) T \mathrm{P}\left[\frac{\left(L_{1}, L_{1} R_{1}\right)}{b_{L}(T)} \in \cdot \stackrel{\mathrm{v}}{\rightarrow} \nu(\cdot)\right.$ on $D$,

where $\nu$ is a homogeneous Radon measure of order $-\alpha_{L}$.

$\left(\mathrm{IIB}^{\prime}\right) T \mathrm{P}\left[\frac{\left(R_{1}, L_{1} R_{1}\right)}{b_{R}(T)} \in \cdot \stackrel{\mathrm{v}}{\rightarrow} \nu(\cdot)\right.$ on $D$,

where $\nu$ is a homogeneous Radon measure of order $-\alpha_{R}$.

Using Corollary 2.1 and conditions (IC) and (IIC), we can extend the multivariate regular variation in the conditions $\left(\mathrm{IB}^{\prime}\right)$ and $\left(\mathrm{IIB}^{\prime}\right)$ to the cone $[\mathbf{0}, \boldsymbol{\infty}] \backslash\{\mathbf{0}\}$.

\section{LÉvy Approximation}

We now give a Lévy approximation to the cumulative input process when input rates are random. Observe from Theorem 3.1 that the product $L_{1} R_{1}$ has a tail of index $\alpha_{P}:=\alpha_{L} \wedge \alpha_{R}$, and hence has a finite mean. Let us call it $\mu_{P}:=\mathrm{E}\left(L_{1} R_{1}\right)$. Then we have the following asymptotic behavior of the process $A(t)$, defined as in (4.1), measured at a large scale.

Theorem 5.1. Assume (4.2)-(4.3) and (IA)-(IC) or (IIA)-(IIC). Define

$$
X^{(T)}(t)=\frac{A(T t)-\lambda T t \mu_{P}}{b_{P}(T)} .
$$

Then

$$
X^{(T)}(\cdot) \stackrel{\text { fidi }}{\rightarrow} X_{\alpha_{P}}(\cdot),
$$

where the above convergence is the usual weak convergence of finite dimensional distributions and $X_{\alpha}$ is a mean 0 , skewness $1, \alpha$-stable Lévy motion with scale parameter $\left(\lambda / C_{\alpha}\right)^{1 / \alpha}$ and

$$
C_{\alpha}=\frac{1-\alpha}{\Gamma(2-\alpha) \cos \left(\frac{\pi \alpha}{2}\right)} .
$$

We shall prove the theorem in two parts. First we prove the one-dimensional convergence and then we prove the finite dimensional convergence for any number of dimensions.

For the analysis, it helps to consider the Poisson point process,

$$
M=\sum_{k=1}^{\infty} \varepsilon_{\left(\Gamma_{k}, L_{k}, R_{k}\right)}
$$

with mean measure $\lambda d t \times F$ on $(0, \infty)^{3}$.

The random variable $A(T)$ is a function of the random measure restricted to $\mathcal{R}^{(T)}=\{(x, y, z) \in$ $\left.(0, \infty)^{3}: x<T\right\}$. It helps to split $\mathcal{R}^{(T)}$ into two disjoint sets

$$
\begin{aligned}
& \mathcal{R}_{1}^{(T)}=\left\{(x, y, z) \in(0, \infty)^{3}: x+y \leqslant T\right\}, \\
& \mathcal{R}_{2}^{(T)}=\left\{(x, y, z) \in(0, \infty)^{3}: x<T<x+y\right\} .
\end{aligned}
$$

The corresponding input processes are

$$
A_{1}(T)=\sum_{k=1}^{\infty} R_{k} L_{k} \mathbf{1}_{\left[\left(\Gamma_{k}, L_{k}, R_{k}\right) \in \mathcal{R}_{1}^{(T)}\right]},
$$




$$
A_{2}(T)=\sum_{k=1}^{\infty} R_{k}\left(T-\Gamma_{k}\right) \mathbf{1}_{\left[\left(\Gamma_{k}, L_{k}, R_{k}\right) \in \mathcal{R}_{2}^{(T)}\right]} .
$$

with $A(T)=A_{1}(T)+A_{2}(T)$. Since $A_{i}(T), i=1,2$ are functions of $\left.M\right|_{\mathcal{R}_{i}^{(T)}}, i=1,2$ respectively, and $\mathcal{R}_{1}(T) \cap \mathcal{R}_{2}(T)=\emptyset$, we have $A_{1}(T)$ and $A_{2}(T)$ are independent.

Now,

$$
\mathrm{E}\left[M\left(\mathcal{R}_{1}^{(T)}\right)\right]=\int_{x=0}^{T} \int_{y \in(0, T-x]} \int_{z=0}^{\infty} \lambda d x F(d y, d z)=\lambda \int_{x=0}^{T} F_{L}(T-x) d x=\lambda \int_{0}^{T} F_{L}(x) d x=: \lambda \widehat{F_{L}}(T),
$$

and, similarly, as $T \rightarrow \infty$,

$$
\mathrm{E}\left[M\left(\mathcal{R}_{2}^{(T)}\right)\right]=\lambda \int_{x=0}^{T} \bar{F}_{L}(x) d x=: \lambda m_{L}(T) \rightarrow \lambda \mu_{L}
$$

where $\mu_{L}=\mathrm{E}\left(L_{1}\right)<\infty$ as $\alpha_{L}>1$. Since $\mathrm{E}\left(M\left(\mathcal{R}_{i}^{(T)}\right)\right)<\infty, i=1,2$, we have the representation

$$
\left.M\right|_{\mathcal{R}_{1}^{(T)}} \stackrel{\mathrm{d}}{=} \sum_{k=1}^{P(T)} \varepsilon_{\left(\tau_{k}^{(T)}, J_{k}^{(T)}, S_{k}^{(T)}\right)}
$$

where $P(T) \sim \operatorname{POI}\left(\lambda \widehat{F}_{L}(T)\right)$; i. e., a Poisson random variable with parameter $\lambda \widehat{F}_{L}(T)$, independent of the iid random vectors

$$
\left.\left(\tau_{k}^{(T)}, J_{k}^{(T)}, S_{k}^{(T)}\right) \sim \frac{d x F(d y, d z)}{\widehat{F_{L}}(T)}\right|_{\mathcal{R}_{1}^{(T)}},
$$

where the above statement means the vector on the left has a distribution given on the right. Similarly

$$
\left.\left.M\right|_{\mathcal{R}_{2}^{(T)}} \stackrel{\mathrm{d}}{=} \sum_{k=1}^{P^{\prime}(T)} \varepsilon_{\left(\tau_{k}^{\prime(T)}, J_{k}^{\prime(T)}, S_{k}^{\prime}\right.}^{(T)}\right),
$$

where $P^{\prime}(T) \sim \operatorname{POI}\left(\lambda m_{L}(T)\right)$ independent of the iid random vectors

$$
\left.\left({\tau_{k}^{\prime}}^{(T)}, J_{k}^{\prime(T)}, S_{k}^{\prime(T)}\right) \sim \frac{d x F(d y, d z)}{m_{L}(T)}\right|_{\mathcal{R}_{2}^{(T)}} .
$$

The key step in the entire analysis is to study the tail behavior of $J_{1}^{(T)} S_{1}^{(T)}$.

Lemma 5.1. Under the assumptions of Theorem 5.1,

$$
T \mathrm{P}\left[\frac{J_{1}^{(T)} S_{1}^{(T)}}{b_{P}(T)}>w\right] \rightarrow w^{-\alpha} \text { as } T \rightarrow \infty,
$$

where the convergence is uniform for $w \in[a, \infty), \forall a>0$.

Proof. We consider Case (I) assumptions only. The analysis in Case (II) is similar and details can be found in the technical report by Maulik et al. [13]. 
Using (5.3) and the fact that $\frac{1}{T} \widehat{F}_{L}(T)=\frac{1}{T} \int_{0}^{T} F_{L}(u) d u \sim F_{L}(T) \rightarrow 1$, we have,

$$
\begin{aligned}
T \mathrm{P}\left[J_{1}^{(T)} S_{1}^{(T)}>b_{L}(T) w\right] & \sim \iint_{\substack{y \in(0, T) \\
y z>b_{L}(T) w}} \int_{y}^{T} d u F(d y, d z)=\int_{0}^{T} \iint_{\substack{y \in(0, u] \\
y z>b_{L}(T) w}} F(d y, d z) d u \\
& =\frac{1}{T} \int_{0}^{T} T \mathrm{P}\left[L_{1} \leqslant u, \frac{L_{1}}{b_{L}(T)} R_{1}>w\right] d u \\
& =\frac{b_{L}(T)}{T} \int_{0}^{\frac{T}{b_{L}(T)}} T \mathrm{P}\left[\frac{\left(L_{1}, L_{1} R_{1}\right)}{b_{L}(T)} \in(0, u] \times(w, \infty)\right] d u .
\end{aligned}
$$

Now, $(0, u] \times(w, \infty)$ is bounded away from $\mathbf{0}$ and hence is relatively compact in $[\mathbf{0}, \infty] \backslash\{\mathbf{0}\}$ and has boundary with $\nu$-measure zero. Hence by the assumptions (IB) or (IC) and using Corollary 2.1, we have, as $T \rightarrow \infty$,

$$
T \mathrm{P}\left[\frac{\left(L_{1}, L_{1} R_{1}\right)}{b_{L}(T)} \in(0, u] \times(w, \infty)\right] \rightarrow \nu((0, u] \times(w, \infty))=\left(\nu_{\alpha_{L}} \times G\right)\left(A_{u, w}\right),
$$

where $A_{u, w}=\{(y, z): y \leqslant u, y z>w\}$ and

$$
\lim _{u \rightarrow \infty}\left(\nu_{\alpha_{L}} \times G\right)\left(A_{u, w}\right)=w^{-\alpha_{L}} \int_{0}^{\infty} u^{\alpha_{L}} G(d u)=: c_{w}<\infty .
$$

Also, the integrand on the right side of (5.6) is bounded above by $T \mathrm{P}\left[L_{1} R_{1}>b_{L}(T) w\right]$, which, by Theorem 3.1, converges to $c_{w}$. Hence, from (5.6), we get,

$$
T \mathrm{P}\left[\frac{J_{1}^{(T)} S_{1}^{(T)}}{b_{L}(T)}>w\right] \rightarrow w^{-\alpha_{L}} \int_{0}^{\infty} u^{\alpha_{L}} G(d u) .
$$

and, hence,

$$
T \mathrm{P}\left[\frac{J_{1}^{(T)} S_{1}^{(T)}}{\tilde{b}(T)}>w\right] \rightarrow w^{-\alpha_{P}}
$$

where $\tilde{b}(T):=\left(\int_{0}^{\infty} u^{\alpha_{P}} G(d u)\right)^{1 / \alpha_{P}} b_{L}(T) \sim b_{P}(T)$ by Theorem 3.1. The left side of (5.7) is monotone non-increasing and the right side is continuous in $(0, \infty)$. Hence, [cf. 22, pg. 1] pointwise convergence implies locally uniform convergence in $(0, \infty)$, and thus

$$
T \mathrm{P}\left[\frac{J_{1}^{(T)} S_{1}^{(T)}}{b_{P}(T)}>w\right] \rightarrow w^{-\alpha_{P}}
$$

Since the left side in (5.8) above is monotone non-increasing with a continuous pointwise limit on $(0, \infty)$ which has a finite limit at $\infty$, the convergence is uniform on $[a, \infty)$.

To complete the proof of Theorem 5.1 for one-dimensional convergence, we need the following lemma studying the moment conditions of $J_{1}^{(T)} S_{1}^{(T)} / b_{P}(T)$, which follows easily using Lemma 5.1 and Karamata's theorem. 
Lemma 5.2. Under the assumptions of Theorem 5.1, we have the following results:

$$
\begin{aligned}
& \lim _{M \rightarrow \infty} \limsup _{T \rightarrow \infty} T \mathrm{E}\left(\frac{J_{1}^{(T)} S_{1}^{(T)}}{b_{P}(T)} \mathbf{1}\left[\frac{J_{1}^{(T)} S_{1}^{(T)}}{b_{P}(T)}>M\right]\right)=0, \\
& \left.\quad \limsup _{T \rightarrow \infty} T \operatorname{Var}\left(\frac{J_{1}^{(T)} S_{1}^{(T)}}{b_{P}(T)} \mathbf{1}^{\left[\frac{J_{1}^{(T)} S_{1}^{(T)}}{b_{P}(T)} \leqslant M\right]}\right]\right) \leqslant \frac{\alpha_{P}}{2-\alpha_{P}} M^{2-\alpha_{P}} \quad \forall M>0,
\end{aligned}
$$

and hence,

$$
\lim _{\varepsilon \downarrow 0} \limsup _{T \rightarrow \infty} T \operatorname{Var}\left(\frac{J_{1}^{(T)} S_{1}^{(T)}}{b_{P}(T)} \mathbf{1}\left[\frac{J_{1}^{(T)} S_{1}^{(T)}}{b_{P}(T)} \leqslant \varepsilon\right]\right)=0 .
$$

Further,

$$
\limsup _{T \rightarrow \infty} b_{P}(T) \mathrm{E}\left(\frac{J_{1}^{(T)} S_{1}^{(T)}}{b_{P}(T)}\right) \leqslant \mathrm{E}\left[L_{1} R_{1}\right]
$$

and hence,

$$
\lim _{T \rightarrow \infty} \mathrm{E}\left(\frac{J_{1}^{(T)} S_{1}^{(T)}}{b_{P}(T)}\right)=0 .
$$

Now, we prove Theorem 5.1 for the process $A_{1}$ defined in (5.1), albeit with a different centering.

Theorem 5.2. Under assumptions (4.2)-(4.3) and (IA)-(IC) or (IIA)-(IIC), we have

$$
\frac{A_{1}(T)-P(T) \mathrm{E}\left(J_{1}^{(T)} S_{1}^{(T)}\right)}{b_{P}(T)} \Rightarrow X_{\alpha_{P}}(1),
$$

where $X_{\alpha_{P}}$ is as defined in Theorem 5.1.

Proof. As in Section 2 of Resnick and Samorodnitsky [20], using (5.9), (5.11), (5.13), we get

$$
S_{T} \Rightarrow S_{\alpha_{P}} \text { in } D([0, \infty)) \text {, }
$$

where

$$
S_{T}(t):=\sum_{k=1}^{\lfloor T t\rfloor}\left[\frac{J_{k}^{(T)} S_{k}^{(T)}}{b_{P}(T)}-\mathrm{E}\left(\frac{J_{k}^{(T)} S_{k}^{(T)}}{b_{P}(T)}\right)\right]
$$

and $S_{\alpha_{P}}$ is an $\alpha_{P}$-Lévy motion with the skewness parameter 1 , mean 0 , and scaling parameter $C_{\alpha_{P}}^{-\frac{1}{\alpha_{P}}}$.

Since $P(T) \sim \operatorname{POI}\left(\lambda \widehat{F}_{L}(T)\right)$ and $\lambda \widehat{F}_{L}(T) \sim \lambda T \rightarrow \infty$, we have $P(T) / T \Rightarrow \lambda$ in $[0, \infty)$. By independence of $S_{T}$ and $P(T) / T$, we have

$$
\left(S_{T}, \frac{P(T)}{T}\right) \Rightarrow\left(S_{\alpha_{P}}, \lambda\right) \text { in } D([0, \infty)) \times[0, \infty) .
$$


Hence, by Whitt [25],

$$
S_{T}\left(\frac{P(T)}{T}\right) \Rightarrow S_{\alpha_{P}}(\lambda) \text { in } \mathbb{R}
$$

Thus,

$$
S_{T}\left(\frac{P(T)}{T}\right)=\frac{A_{1}(T)}{b_{P}(T)}-P(T) \mathrm{E}\left(\frac{J_{1}^{(T)} S_{1}^{(T)}}{b_{P}(T)}\right) \Rightarrow S_{\alpha_{P}}(\lambda) \text { in } \mathbb{R} .
$$

Note, $S_{\alpha_{P}}(\lambda)$ is $\alpha_{P}$-stable random variable with skewness parameter 1 , mean 0 and scaling parameter $\left(\lambda / C_{\alpha_{P}}\right)^{1 / \alpha_{P}}$ and hence has same distribution as $X_{\alpha_{P}}(1)$, and the result is proved.

We prove $A_{2}$ is negligible in the following theorem.

Theorem 5.3. If $A_{2}$ is defined as in (5.2), then

$$
\frac{A_{2}(T)}{b_{P}(T)} \stackrel{P}{\rightarrow} 0
$$

Proof. Again, we consider Case (I) only. The details of the proof of Case (II), which are similar, can be found in Maulik et al. [13]. It is enough to show $\frac{A_{2}(T)}{b_{L}(T)} \stackrel{P}{\rightarrow} 0$, since $\frac{b_{P}(T)}{b_{L}(T)}$ converges to a constant, which is positive and finite.

Fix $\varepsilon>0, \eta>0$. Observe that $P^{\prime}(T) \sim \operatorname{POI}\left(\lambda m_{L}(T)\right)$ and $m_{L}(T) \rightarrow \mu_{L}$. Thus, $P^{\prime}(T) \Rightarrow P^{\prime}$, where $P^{\prime} \sim \operatorname{POI}\left(\lambda \mu_{L}\right)$. Choose $M$ such that $\mathrm{P}\left[P^{\prime}>M\right]<\frac{\varepsilon}{2}$. Then for all large enough $T$, we have $\mathrm{P}\left[P^{\prime}(T)>M\right]<\frac{\varepsilon}{2}$ and

$$
\begin{aligned}
\mathrm{P}\left[\frac{A_{2}(T)}{b_{L}(T)}>\eta\right] & \leqslant \mathrm{P}\left[\frac{A_{2}(T)}{b(T)}>\eta, P^{\prime}(T) \leqslant M\right]+\mathrm{P}\left[P^{\prime}(T)>M\right] \\
& \leqslant \mathrm{P}\left[\sum_{k=1}^{M}\left(T-\tau_{k}^{\prime(T)}\right) \frac{S_{k}^{\prime(T)}}{b_{L}(T)}>\eta\right]+\frac{\varepsilon}{2} \leqslant M \mathrm{P}\left[\left(T-\tau_{1}^{\prime(T)}\right) \frac{S_{1}^{\prime(T)}}{b_{L}(T)}>\frac{\eta}{M}\right]+\frac{\varepsilon}{2} .
\end{aligned}
$$

Thus, it is enough to show that $\left(T-\tau_{1}^{\prime(T)}\right) S_{1}^{(T)} / b(T)$ converges to 0 in probability. Now, by (5.4), we have,

$$
\mathrm{P}\left[T-\tau_{1}^{\prime(T)} \leqslant s\right]=\mathrm{P}\left[\tau_{1}^{\prime(T)} \geqslant T-s\right]=\frac{\int_{x=T-s}^{T} \bar{F}_{L}(T-x) d x}{m_{L}(T)}=\int_{0}^{s} \frac{\bar{F}_{L}(x)}{m_{L}(T)} d x .
$$

Thus, $T-\tau_{1}^{\prime(T)}$ has density $\bar{F}_{L}(\cdot) / m_{L}(T)$, supported on $(0, T)$, which converges pointwise to a density function $\bar{F}_{L}(\cdot) / \mu_{L}$, supported on $\mathbb{R}_{+}$. Hence, by Scheffé's theorem, $T-\tau_{1}^{\prime(T)}$ converges weakly to a positive random variable with density $\bar{F}_{L}(\cdot) / \mu_{L}$. So it is enough to show, by Slutsky's theorem, that $S_{1}^{\prime(T)} / b(T)$ is negligible in probability.

Fix $\eta>0$. Now observe, by (5.4),

$$
\begin{aligned}
\mathrm{P}\left[\frac{S_{1}^{\prime}(T)}{b_{L}(T)}>\eta\right] & =\frac{1}{m_{L}(T)} \int_{0}^{T} \mathrm{P}\left[L_{1}>T-x, \frac{R_{1}}{b_{L}(T)}>\eta\right] d x=\frac{1}{m_{L}(T)} \int_{0}^{T} \mathrm{P}\left[L_{1}>x, \frac{R_{1}}{b_{L}(T)}>\eta\right] d x \\
& \leqslant \frac{1}{m_{L}(T)} T \mathrm{P}\left[\frac{R_{1}}{b_{L}(T)}>\eta\right]=\frac{1}{m_{L}(T)} T \mathrm{P}\left[\frac{R_{1}}{b_{R}(T)}>\frac{b_{L}(T)}{b_{R}(T)} \eta\right] \rightarrow 0
\end{aligned}
$$


since $\frac{b_{L}(T)}{b_{R}(T)} \in R V_{\frac{1}{\alpha_{L}}-\frac{1}{\alpha_{R}}}$ and $\alpha_{L}<\alpha_{R}$ imply $\frac{b_{L}(T)}{b_{R}(T)} \rightarrow \infty$ and $m_{L}(T) \rightarrow \mu_{L}<\infty$. Therefore, $S_{1}^{(T)} / b_{L}(T) \stackrel{P}{\rightarrow} 0$.

Combining (5.14) and (5.15) and using Slutsky's theorem, we get Theorem 5.1 for one-dimensional convergence with a random centering:

$$
\frac{A(T)}{b_{P}(T)}-P(T) \mathrm{E}\left(\frac{J_{1}^{(T)} S_{1}^{(T)}}{b_{P}(T)}\right) \Rightarrow X_{\alpha_{P}}(1)
$$

We now obtain the correct centering. Observe that we need to have the centering $\frac{\lambda T \mu_{P}}{b_{P}(T)}$ in place of $P(T) \mathrm{E}\left[\frac{J_{1}^{(T)} S_{1}^{(T)}}{b_{P}(T)}\right]$ in (5.16) to get the required result. We shall show the difference of the above two expressions goes to 0 in probability. Now,

$$
\begin{aligned}
& P(T) \mathrm{E}\left(\frac{J_{1}^{(T)} S_{1}^{(T)}}{b_{P}(T)}\right)-\frac{\lambda T \mu_{P}}{b_{P}(T)} \\
= & \frac{P(T)-\lambda \widehat{F}_{L}(T)}{\sqrt{\lambda \widehat{F}_{L}(T)}} \sqrt{\lambda \widehat{F}_{L}(T)} \mathrm{E}\left(\frac{J_{1}^{(T)} S_{1}^{(T)}}{b_{P}(T)}\right)-\frac{\lambda}{b_{P}(T)}\left[T \mu_{P}-\widehat{F}_{L}(T) \mathrm{E}\left(J_{1}^{(T)} S_{1}^{(T)}\right)\right] .
\end{aligned}
$$

Now, as in (5.12),

$$
\limsup _{T \rightarrow \infty} \sqrt{\lambda \widehat{F}_{L}(T)} \mathrm{E}\left(\frac{J_{1}^{(T)} S_{1}^{(T)}}{b_{P}(T)}\right) \leqslant \lim _{T \rightarrow \infty} \frac{\lambda \widehat{F}_{L}(T)}{b_{P}(T)} \mathrm{E}\left[L_{1} R_{1}\right]=\lim _{T \rightarrow \infty} \frac{\sqrt{T \lambda}}{b_{P}(T)} \mathrm{E}\left(L_{1} R_{1}\right) \rightarrow 0,
$$

since $\frac{\sqrt{T}}{b_{P}(T)} \in R V_{\frac{1}{2}-\frac{1}{\alpha_{P}}}$ and $\alpha_{P}<2$. Since $\widehat{F}_{L}(T) \sim T$, using the central limit theorem, we have $\left(P(T)-\lambda \widehat{F}_{L}(T)\right) / \lambda \widehat{F}_{L}(T)^{1 / 2}$ is bounded in probability and we get the first term on the right side of (5.17) goes to 0 in probability. Thus, we only need to show the second term on the right side of (5.17), which is just a number, goes to zero. Observe, from (5.3),

$$
\begin{aligned}
T \mu_{P} & -\widehat{F}_{L}(T) \mathrm{E}\left(J_{1}^{(T)} S_{1}^{(T)}\right)=\int_{x=0}^{T} \iint_{(y, z) \in \mathbb{R}_{+}^{2}} y z F(d y, d z) d x-\int_{x=0}^{T} \int_{y \leqslant T-x} \int_{z \in(0, \infty)} y z F(d y, d z) d x \\
& =\int_{x=0}^{T} \int_{y>T-x} \int_{z \in(0, \infty)} y z F(d y, d z) d x=\int_{x=0}^{T} \int_{y>x} \int_{z \in(0, \infty)} y z F(d y, d z) d x \\
& =\int_{x=0}^{T}\left[\int_{y>x} \int_{z \in(0,1]} y z F(d y, d z)+\int_{y>x} \int_{z>1} y z F(d y, d z)\right] d x \\
\leqslant & \int_{x=0}^{T}\left[\int_{y>x} y F_{L}(d y)+\int_{u>x} u F_{P}(d u)\right] d x
\end{aligned}
$$


where $L_{1} R_{1}$ has distribution $F_{P}$. Then, by (5.18) and (5.19), we get, $(5.20)$

$0 \leqslant \frac{\lambda}{b_{P}(T)}\left[T \mu_{P}-\widehat{F}_{L}(T) \mathrm{E}\left(J_{1}^{(T)} S_{1}^{(T)}\right)\right] \leqslant \frac{\lambda}{b_{P}(T)} \int_{x=0}^{T} \int_{y>x} y F_{L}(d y) d x+\frac{\lambda}{b_{P}(T)} \int_{x=0}^{T} \int_{u>x} u F_{P}(d u) d x$.

Now, using Karamata's theorem, we get,

$$
\frac{\lambda}{b_{P}(T)} \int_{x=0}^{T} \int_{y>x} y F_{L}(d y) d x \sim \frac{\lambda}{\left(2-\alpha_{L}\right)\left(\alpha_{L}-1\right)} \frac{T^{2} \bar{F}_{L}(T)}{b_{P}(T)} \rightarrow 0
$$

since it is a regularly varying function of index $2-\alpha_{L}-\alpha_{P}{ }^{-1} \leqslant 2-\alpha_{P}-\alpha_{P}{ }^{-1}=-\left(\alpha_{P}-1\right)^{2} / \alpha_{P}<0$. Similarly,

$$
\frac{\lambda}{b_{P}(T)} \int_{x=0}^{T} \int_{u>x} u F_{P}(d u) d x \sim \frac{\lambda}{\left(2-\alpha_{P}\right)\left(\alpha_{P}-1\right)} \frac{T^{2} \overline{F_{P}}(T)}{b_{P}(T)} \in R V_{2-\alpha_{P}-\frac{1}{\alpha_{P}}}
$$

and hence goes to zero. Thus, by (5.20),

$$
\frac{\lambda}{b_{P}(T)}\left[T \mu_{P}-\widehat{F}_{L}(T) \mathrm{E}\left(J_{1}^{(T)} S_{1}^{(T)}\right)\right] \rightarrow 0 .
$$

Thus, on $\mathbb{R}$,

$$
\frac{A(T)-\lambda T \mu_{P}}{b_{P}(T)} \Rightarrow X_{\alpha_{P}}(1)
$$

so that for all $t \geqslant 0$,

$$
\frac{A(T t)-\lambda T t \mu_{P}}{b_{P}(T)}=\frac{b_{P}(T t)}{b_{P}(T)} \frac{A(T t)-\lambda T t \mu_{P}}{b_{P}(T t)} \Rightarrow t^{\frac{1}{\alpha_{P}}} X_{\alpha_{P}}(1) \stackrel{\mathrm{d}}{=} X_{\alpha_{P}}(t) .
$$

This is the required one-dimensional convergence:

$$
\frac{A(T t)-\lambda T t \mu_{P}}{b_{P}(T)} \Rightarrow X_{\alpha_{P}}(t) \text { in } \mathbb{R} \quad \forall t \geqslant 0 .
$$

Finally, we consider the finite dimensional convergence, which will complete the proof of Theorem 5.1. Let $0<s<t$. Observe that the process $A_{1}(T \cdot)$ has independent increments. Also, let us define,

$$
A_{1}(T t)-A_{1}(T s)=B_{T}(s, t)+C_{T}(s, t),
$$

where

$$
B_{T}(s, t)=\iiint_{\substack{0 \leqslant x \leqslant T s \\ T s<x+y \leqslant T t}} y z M(d x, d y, d z,) \text { and } C_{T}(s, t)=\iiint_{\substack{T s<x \leqslant T t \\ T s<x+y \leqslant T t}} y z M(d x, d y, d z) .
$$

It is easy to check that $C_{T}(s, t) \stackrel{\mathrm{d}}{=} A_{1}(T(t-s))$. So, by (5.22), we get,

$$
\frac{C_{T}(s, t)-\lambda T(t-s) \mu_{P}}{b_{P}(T)} \Rightarrow X_{\alpha_{P}}(t-s) \stackrel{\mathrm{d}}{=} X_{\alpha_{P}}(t)-X_{\alpha_{P}}(s) .
$$


Also, by (5.18) and (5.21), we get,

$$
\begin{aligned}
\mathrm{E}\left(\frac{B_{T}(s, t)}{b_{P}(T)}\right) & =\frac{\lambda}{b_{P}(T)} \iiint_{\substack{0 \leqslant x \leqslant T s \\
T s<x+y \leqslant T t}} y z F(d y, d z) d x \leqslant \frac{\lambda}{b_{P}(T)} \iiint_{\substack{0 \leqslant x \leqslant T s \\
x+y>T s}} y z F(d y, d z) d x \\
& =\frac{b_{P}(T s)}{b_{P}(T)} \lambda \frac{T s \mu_{P}-\widehat{F}_{L}(T s) \mathrm{E}\left(J_{1}^{(T s)} S_{1}^{(T s)}\right)}{b_{P}(T s)} \rightarrow s^{-\alpha_{P}} \cdot 0,
\end{aligned}
$$

which implies

$$
\frac{B_{T}(s, t)}{b_{P}(T)} \stackrel{P}{\rightarrow} 0
$$

Then, by $(5.23)-(5.25)$,

$$
\frac{\left(A_{1}(T t)-\lambda T t \mu_{P}\right)-\left(A_{1}(T s)-\lambda T s \mu_{P}\right)}{b_{P}(T)} \Rightarrow X_{\alpha_{P}}(t)-X_{\alpha_{P}}(s) \text { in } \mathbb{R} .
$$

By the independent increment property of $X_{1}^{(T)}$ and $X_{\alpha_{P}}$, coordinatewise convergence of increments of $X_{1}^{(T)}$ and $X_{\alpha_{P}}$ implies joint convergence of increments. Thus, $X_{1}^{(T)} \stackrel{\text { fidi }}{\rightarrow} X_{\alpha_{P}}$. Also, from (5.15), we have, $\left(A_{2}\left(T t_{1}\right), \cdots, A_{2}\left(T t_{k}\right)\right) / b_{P}(T) \stackrel{P}{\rightarrow} \mathbf{0}$, for all $0 \leqslant t_{1}<\cdots<t_{k}$. Now, $X^{(T)}(t)=\frac{A(T t)-\lambda T t \mu_{P}}{b_{P}(T)}=$ $\frac{A_{1}(T t)-\lambda T t \mu_{P}}{b_{P}(T)}+\frac{A_{2}(T t)}{b_{P}(T)}$ implies, $X^{(T)} \stackrel{\text { fidi }}{\rightarrow} X_{\alpha_{P}}$.

\section{Conclusion}

Our result is dependent on the modelling decisions for the joint distribution of $(L, R)$. If our model of asymptotic independence holds, then so does the classical asymptotic independence model. However, our estimate of the spectral distribution function, defined in (2.3), for the time and the rate of transmission [cf. 5, Sections 4 and 5], given in Figure 3, does not seem to be supported on $\left\{0, \frac{\pi}{2}\right\}$, and may suggest a lack of asymptotic independence between the two random variables. So our model, though successful in accommodating a random transmission rate, may not fit this particular data well. The possible failure, as pointed out by the referee, may be due to the assumption of asymptotic independence of the transmission length and the transmission rate. It is more realistic to consider asymptotic independence between the transmission rate and the file size. We address this issue in a later paper.

\section{REFERENCES}

[1] B. Basrak. The sample autocorrelation function of non-linear time series. PhD thesis, Rijksuniversiteit Groningen, Groningen, Netherlands, 2000.

[2] Patrick Billingsley. Convergence of Probability Measures. John Wiley \& Sons Inc., New York, second edition, 1999. ISBN 0-471-19745-9. A Wiley-Interscience Publication.

[3] L. Breiman. On some limit theorems similar to the arc-sin law. Theory Probab. Appl., 10:323-331, 1965.

[4] Carlos A. Cunha, Azer Besavros, and Mark E. Crovella. Characteristics of www client-based traces. Preprint available as Technical Report TR-95-010, Computer Science Department, Boston University at http://www.cs.bu.edu/techreports/pdf, 1995.

[5] Laurens de Haan and John de Ronde. Sea and wind: multivariate extremes at work. Extremes, 1(1): 7-45, 1998. ISSN 1386-1999.

[6] A. Erramilli, O. Narayan, and W. Willinger. Experimental queueing analysis with long-range dependent packet traffic. IEEE/ACM Transactions on Network Computing, 4:209-223, 1996. 


\section{Spectral Distribution Function}

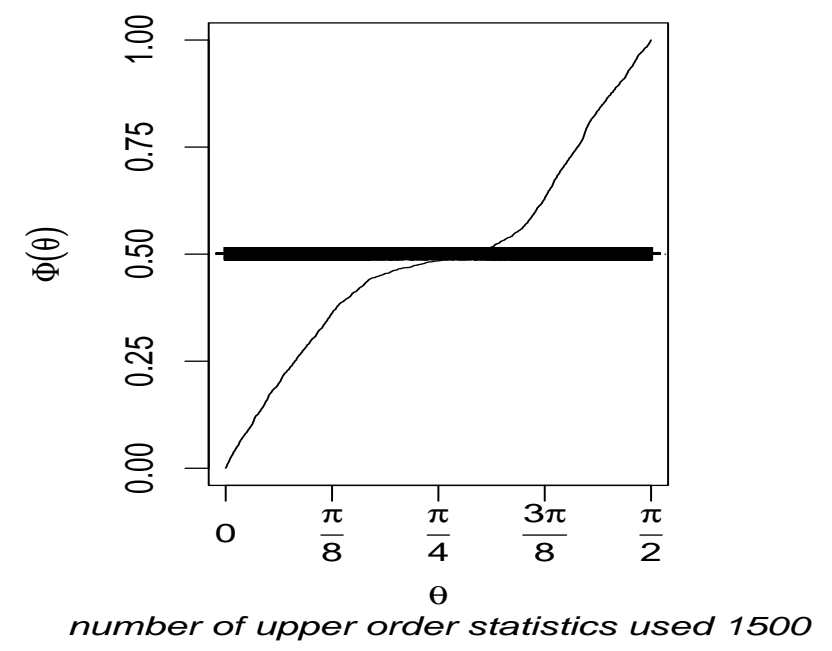

FiguRE 3. Spectral measure estimates of time and rate of transmission

[7] C. A. Guerin, H. Nyberg, O. Perrin, S. Resnick, H. Rootzen, and C. Starica. Empirical testing of the infinite source poisson data traffic model. Technical Report 1257, School of ORIE, Cornell University, Ithaca NY 14853; available at http://www.orie.cornell.edu/trlist/trlist.html., 1999.

[8] D. Heath, S. Resnick, and G. Samorodnitsky. How system performance is affected by the interplay of averages in a fluid queue with long range dependence induced by heavy tails. Ann. Appl. Probab., 9: 352-375, 1999.

[9] P. Jelenković and A. Lazar. Asymptotic results for multiplexing subexponential on-off processes. Advances in Applied Probability, 31:394-421, 1999.

[10] Predrag R. Jelenković and Aurel A. Lazar. A network multiplexer with multiple time scale and subexponential arrivals. In Glasserman Paul, Karl Sigman, and David D. Yao, editors, Stochastic Networks: Stability and Rare Events, volume 117 of Lecture Notes in Statistics, pages 215-235. Springer, New York, 1996.

[11] Takis Konstantopoulos and Si-Jian Lin. Macroscopic models for long-range dependent network traffic. Queueing Systems Theory Appl., 28(1-3):215-243, 1998. ISSN 0257-0130.

[12] Joshua B. Levy and Murad S. Taqqu. Renewal reward processes with heavy-tailed inter-renewal times and heavy-tailed rewards. Bernoulli, 6(1):23-44, 2000. ISSN 1350-7265.

[13] Krishanu Maulik, Sidney Resnick, and Holger Rootzén. A network traffic model with random transmission rate. Technical report 1278, available at http://www.orie.cornell.edu/trlist/trlist.html, 2000.

[14] Thomas Mikosch, Sidney Resnick, Holger Rootzén, and Alwin Stegeman. Is network traffic approximated by stable Lévy motion or fractional Brownian motion? Ann. Appl. Probab., 12(1):23-68, 2002. ISSN 1050-5164.

[15] Vladas Pipiras and Murad S. Taqqu. The limit of a renewal reward process with heavy-tailed rewards is not a linear fractional stable motion. Bernoulli, 6(4):607-614, 2000. ISSN 1350-7265.

[16] Vladas Pipiras, Murad S. Taqqu, and Joshua B. Levy. Slow and fast growth conditions for renewal reward processes with heavy tailed renewals and either finite-variance or heavy tailed rewards. PREPRINT, 2000.

[17] S. Resnick. Heavy tail modeling and teletraffic data. Ann. Statist., 25:1805-1869, 1997.

[18] S. Resnick and C. Stărică. Smoothing the Hill estimator. Adv. Appl. Probab., 29:271-293, 1997. 
[19] Sidney Resnick and Holger Rootzén. Self-similar communication models and very heavy tails. Ann. Appl. Probab., 10(3):753-778, 2000. ISSN 1050-5164.

[20] Sidney Resnick and Gennady Samorodnitsky. A heavy traffic approximation for workload processes with heavy tailed service requirements. Management Science, 46(9):1236-1248, 2000.

[21] Sidney Resnick and Eric van den Berg. Weak convergence of high-speed network traffic models. J. Appl. Probab., 37(2):575-597, 2000. ISSN 0021-9002.

[22] Sidney I. Resnick. Extreme values, regular variation, and point processes. Springer-Verlag, New York, 1987. ISBN 0-387-96481-9.

[23] A. V. Skorohod. Limit theorems for stochastic processes. Theory of Probability and Its Applications, 1 (3):261-290, 1956.

[24] Cătălin Stărică. Multivariate extremes for models with constant conditional correlations. J. Empirical Finance, 6:515-553, 1999.

[25] Ward Whitt. Some useful functions for functional limit theorems. Math. Oper. Res., 5(1):67-85, 1980. ISSN 0364-765X.

[26] Ward Whitt. Limits for cumulative input processes to queues. Preprint, AT\&T Labs, 1999.

[27] Ward Whitt. On the Skorohod $M$-topologies. Preprint, AT\&T Labs, 1999.

[28] Ward Whitt. The reflection map is Lipschitz with appropriate Skorohod $M$-metrics. Preprint, AT\&T Labs, 1999.

[29] Ward Whitt. Stochastic-process limits. An introduction to stochastic-process limits and their application to queues. Springer-Verlag, New York, 2002. ISBN 0-387-95358-2.

[30] W. Willinger and V. Paxson. Where mathematics meet the internet. Notices of American Mathematical Society, 45(8):961-970, 1998.

[31] W. Willinger, M. S. Taqqu, M. Leland, and D. Wilson. Self-similarity in high-speed packet traffic: analysis and modelling of ethernet traffic measurements. Statistical Science, 10:67-85, 1995.

[32] Walter Willinger, Murad S. Taqqu, Robert Sherman, and Daniel V. Wilson. Self-similarity through high variability: statistical analysis of Ethernet LAN traffic at the source level (extended version). IEEE/ACM Transactions on Networking, 5(1):71-86, 1997.

Department of Statistical Science, Cornell University, Ithaca, NY 14853, USA

E-mail address: km75@cornell.edu

School of Operations Research and Industrial Engineering, Cornell University, IthacA, NY 14853 , USA

E-mail address: sid@orie.cornell.edu

Department of Mathematics, Chalmers University of Technology, S-412 96 Gothenburg, Sweden

E-mail address: rootzen@math.chalmers.se 